\title{
ASSOCIAÇÕES DE PERCEVEJOS MIRÍDEOS (HEMIPTERA: MIRIDAE) COM PLANTAS NO BRASIL
}

Bárbara Cristina Félix Nogueira1*, Lívia Aguiar Coelho², David dos Santos Martins², Bárbara Duarte Barcellos ${ }^{1}$, Sirlene Rodrigues Sartori', Paulo Sérgio Fiuza Ferreira ${ }^{1,2}$.

${ }^{1}$ Departamento de Biologia Animal, Universidade Federal de Viçosa, Viçosa, Minas Gerais, Brasil. ${ }^{2}$ Departamento de Entomologia, Universidade Federal de Viçosa, Viçosa, Minas Gerais, Brasil. * barbaracfn28@gmail.com

\section{RESUMO}

Os mirídeos têm papel importante sobre a economia brasileira devido à sua influência sobre diversas culturas agrícolas. Devido a isso, este artigo foi desenvolvido visando apresentar as espécies de Miridae que possuem associações ou potenciais associações com plantas no Brasil. Para isso, foram realizadas consultas de artigos, livros e coleções de museus. Ao todo, foram encontradas 168 espécies de mirídeos associadas a plantas; estes dados foram manipulados para a elaboração de gráficos representando as interações entre as espécies de percevejos e as plantas hospedeiras no Brasil. As famílias botânicas Poaceae, Asteraceae, Fabaceae e Solanaceae apresentaram mais espécies de mirídeos associadas e incluem importantes culturas para a economia do país. Com base nestas associações, é possível contribuir para ampliar o conhecimento sobre a biologia e o comportamento alimentar de mirídeos, além de fornecer informações sobre o impacto que podem gerar nos sistemas de produção agrícola no Brasil.

Palavras-chave: Insecta, planta hospedeira, fitófagos. 


\section{ABSTRACT \\ ASSOCIATIONS OF PLANT BUGS (HEMIPTERA: MIRIDAE) WITH PLANTS IN BRAZIL.}

The plant bugs play an important role in the Brazilian economy due to their influence on several agricultural crops. Due to this, this article was developed in order to present the species of Miridae that have associations or potential associations with plants in Brazil. For this, we consulted articles, books and collections of museums. In all, 168 species of plant bugs associated to plants were found; these data were manipulated to the elaboration of graphs representing the interactions between the plant bug species and the host plants in Brazil. The botanical families Poaceae, Asteraceae, Fabaceae and Solanaceae presented more species of associated plant bugs and include important crops for the economy of the country. Based on these associations, it is possible to contribute to broadening the knowledge about biology and the feeding behavior of plant bugs, as well as providing information on the impact they can generate in Brazilian agricultural production systems.

Key words: Insecta, host plant, phytophagous insect.

A família Miridae corresponde a $25 \%$ dos Heteroptera (HENRY, 2009), com aproximadamente 11.139 espécies descritas (SCHUH, 2002-2013). A biologia dessa família é praticamente desconhecida na região neotropical e a maioria dos estudos abordam os mirídeos que apresentam importância econômica (FERREIRA, 1999). No Brasil existem 1.084 espécies de Miridae (FERREIRA et al., 2018), sendo que as regiões Norte e Nordeste são as mais carentes de informações acerca desta família (FERREIRA, 1999).

No geral, entre os mirídeos com registro de ocorrência em plantas, $60 \%$ ocorrem em determinada espécie e menos de $20 \%$ possuem associações com mais de dois hospedeiros (CASSIS; SCHUH, 2012). O elevado número de associações de mirídeos e plantas pode ser justificado por mudanças nas práticas agrícolas e introdução de espécies de plantas exóticas (WHEELER, 2001), o que pode ser preocupante visto que, embora os heteroptera não tenham importância significativa na transmissão de doenças para plantas, seu modo de alimentação e tecido alvo permite a transmissão de vetores de patógenos (CARTER, 1973).

Deste modo, o objetivo deste estudo foi ampliar o conhecimento das associações de mirídeos com plantas no Brasil, enfatizando famílias de importância econômica seja na agricultura, medicina ou ornamentação. 
Os dados de associações de Miridae no Brasil foram obtidos por meio dos catálogos: On-line Systematic Catalog of Plant Bugs (Insecta: Heteroptera: Miridae) (SCHUH 20022013) e Catálogo Taxônomico da Fauna do Brasil, assim como em artigos publicados em revistas científicas, livros, dados de rótulos de espécimes depositados no Museu Regional de Entomologia da Universidade Federal de Viçosa (UFVB), Viçosa, MG, no Museu Nacional da Universidade Federal do Rio de Janeiro (MNRJ), Rio de Janeiro, $\mathrm{RJ}$, e de exemplares enviados ao Museu Regional de Entomologia da Universidade Federal de Viçosa para identificação, provenientes de várias localidades do país. As associações com ocorrência em outros países foram consideradas como potenciais associações quando observado a possibilidade de ocorrência no Brasil, devido ao país abrigar as espécies de mirídeo e planta envolvidas na associação. A nomenclatura botânica seguiu a plataforma Catalogue of Life (2017).

Os dados foram organizados em uma matriz básica para análise utilizando o software Microsoft Excel para elaboração dos gráficos que representam as diferentes relações entre espécies de mirídeos com suas plantas associadas.

Foram encontradas 168 espécies de Miridae, distribuídas em 15 tribos, com associação com plantas. Aproximadamente $48 \%$ das espécies de Miridae apresentaram associação com apenas uma planta (Fig.1). Algumas espécies de mirídeos apresentaram mais que trinta associações, como Taylorilygus apicalis (Fieber) com 88 associações pertencentes a 20 famílias; Microtechnites bractatus (Say) com 45 associações em 17 famílias e Collaria oleosa (Distant) com 36 associações em seis famílias de plantas.

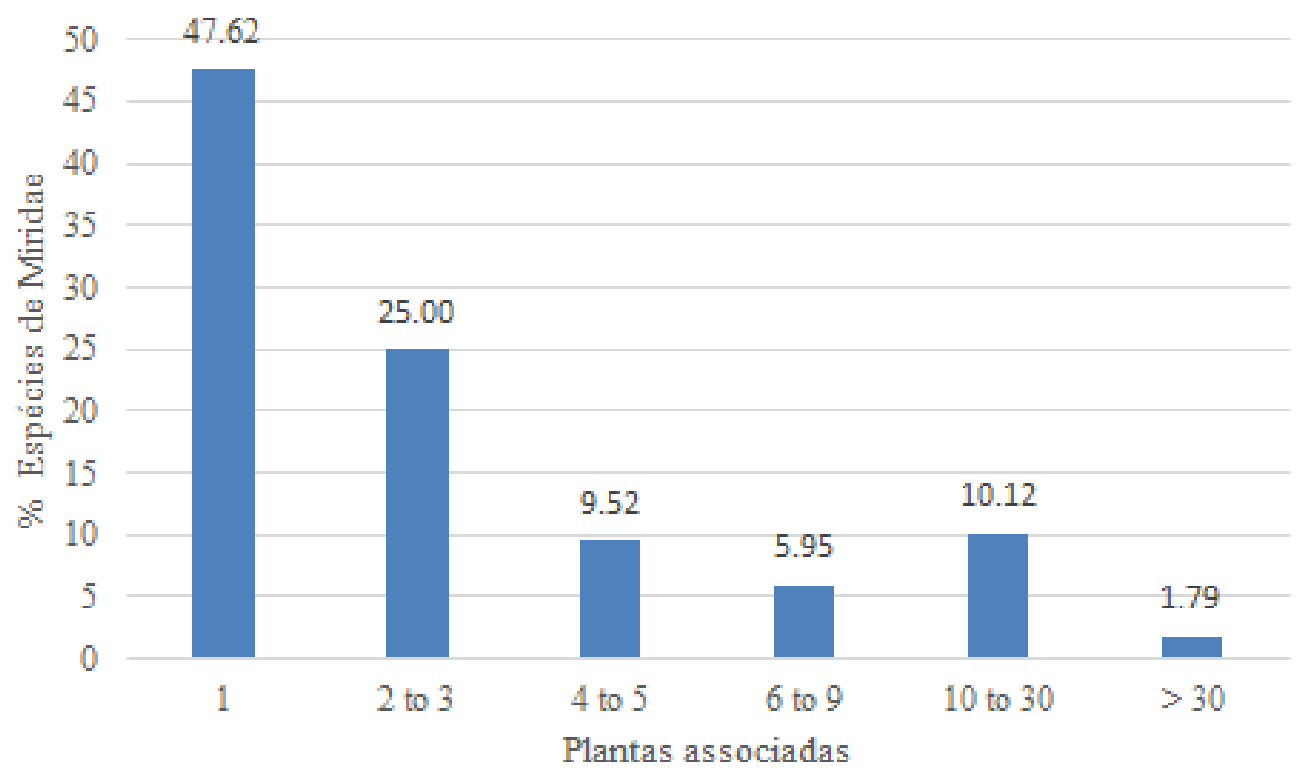

Figura 1. Histograma da porcentagem de espécies de Miridae pelo número de plantas associadas. 
Bárbara Cristina Félix Nogueira, Lívia Aguiar Coelho, David dos Santos

As famílias Poaceae, Asteraceae, b9ém correspondem as famílias onde Fabaceae e Solanaceae foram as que estão inseridas a maioria das culturas apresentaram maior número de asso- importantes economicamente para 0 ciações com espécies de Miridae e tam- país (Fig. 2).

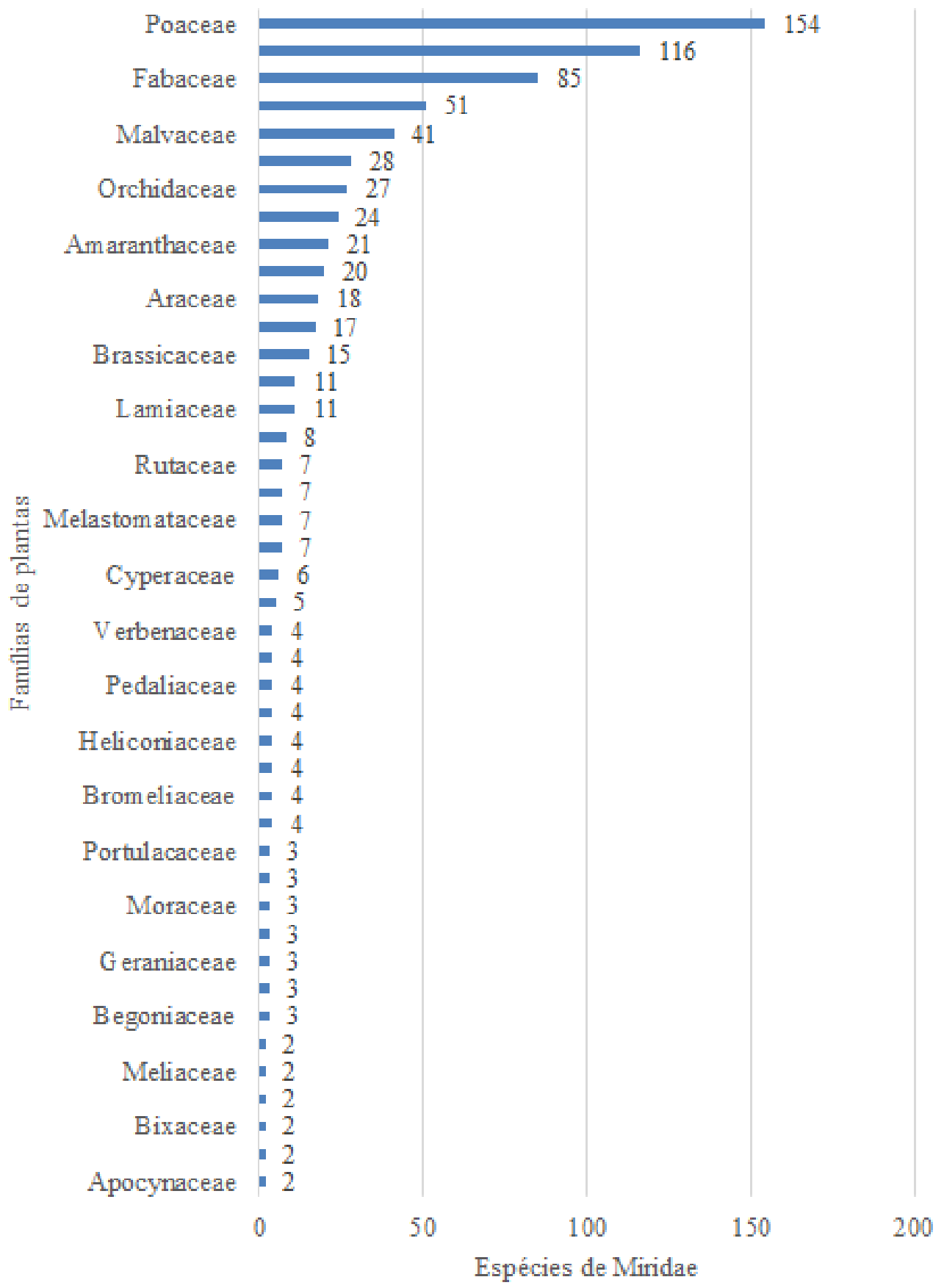

Figura 2. Histograma do número de espécies de Miridae pela família das plantas associadas. Somente foram incluídas famílias de plantas com 2 ou mais espécies de Miridae associados. 
A família Poaceae se destacou por apresentar grande número de associações com mirídeos (Fig. 2) e é extremamente importante para a economia brasileira pois engloba pastagens importantes para o setor pecuário. Neste contexto, é importante ressaltar a existência de relevantes estudos sobre espécies do gênero Collaria da tribo Stenodemini atuando sobre espécies de plantas da família Poaceae (COSTA, 1958; KALVELAGE, 1988; GASSEN, 1996). Além disso, espécies de mirídeos deste gênero são amplamente distribuídos pela América e são consideradas pragas economicamente importantes devido a associação com espécies forrageiras (MELO et al., 2004).

Dentre as famílias de plantas mais utilizadas na medicina alternativa, as mais comuns são Asteraceae, Fabaceae, Solanaceae e Lamiaceae, que estão entre as famílias com maior número de espécies de insetos (GRANDI et al., 1989). Além disso, no âmbito do aspecto ornamental, os gêneros Tenthecoris e Fulvius, chamaram a atenção por apresentarem a maioria dos registros com Orchidaceae.

Em geral, o conhecimento destas relações pode servir de base para novos estudos, contribui para o conhecimento da biologia, associações, comportamento alimentar de mirídeos e fornecem informações sobre possíveis impactos no sistema de produção brasileiro.

\section{Records and distribution}

\section{Briocorinae}

\section{Bryocorini}

Monalocoris carioca Carvalho \& Gomes, 1971: Solanaceae: Ipomoea batatas (L.) Lam. (BR: MG) (UFVB; Ferreira; Rossi, 1979).

Campyloneuropsis cincticornis (Stal, 1860): Fabaceae: Phaseolus vulgaris L. (NI) (Maes; Carvalho, 1989); Solanaceae: Nicotiana tabacum L. (BR: BA) (Silva et al., 1968).

Campyloneuropsis infumatus (Carvalho, 1947): Asteraceae: Calendula officinalis L. (BR; CU) (Martínez et al., 2014); Poaceae: Brachiaria decumbens Stapf. (BR: ES) (Ruiz, 2014); Solanaceae: Nicotiana tabacum L. (BR: MG, SC) (UFVB; Silva et al., 1968; Ferreira; Rossi 1979; Ferreira et al., 2001), Petunia sp. (BR: MG) (Ferreira et al., 2001), Solanum lycopersicum L. (BR) (Martínez et al., 2014).

Campyloneuropsis nigroculatus (Carvalho, 1947): Fabaceae: Senna alexandrina Mill. (BR: MG) (Silva et al., 1968; Ferreira et al., 2001).

Engytatus aristidesi (Carvalho, 1975): Cleomaceae: Tarenaya spinosa (Jacq.) Rafin. (BR: PE) (Carvalho, 1975).

Engytatus modestus (Distant, 1893): Amaranthaceae: Amaranthus sp. (PR) (Capriles, 1969); Apiaceae: Daucus carota L. (BR: BA) (Silva et al., 1968); Asteraceae: Chrysantemum sp. (Wheeler, 2001), Mikania congesta DC. (PR) (Ca- 
priles, 1969); Cleomaceae: Tarenaya spinosa (Jacq.) Rafin. (BR: PE) (Silva et al., 1968; Carvalho, 1975; Maes; Carvalho, 1989); Cucurbitaceae: Cucumis sp. (NI) (Maes; Carvalho, 1989); Fabaceae: Phaseolus vulgaris L.(NI) (Maes; Carvalho, 1989); Liliaceae: Lilium candidum L. (BR: BA) (Silva et al., 1968); Poaceae: Zea mays L. (NI) (Maes; Carvalho, 1989); Portulacaceae: Portulaca sp. (NI) (Maes; Carvalho, 1989); Solanaceae: Nicotiana tabacum L. (PR; NI; BR: BA) (Silva et al., 1968; Capriles, 1969; Maes; CarvaIho, 1989; Wheeler, 2001), Solanum incarceratum Ruiz \& Pav. (BR: BA) (Silva et al., 1968), Solanum lycopersicum L. (CU) (Wheeler, 2001; Hernandez; Henry, 2010), Solanum sisymbriifolium Lam. (BR: BA) (Silva et al., 1968), Solanum tuberosum L. (BR: BA) (Silva et al., 1968), Solanum variabile Mart. (BR: BA) (Silva et al., 1968).

Engytatus varians (Distant, 1884): Amaranthaceae: Amaranthus sp. (CU) (Hernandez; Henry, 2010); Asteraceae: Helianthus annuus L. (CU) (Hernandez; Henry, 2010); Convolvulaceae: Ipomoea batatas (L.) Lam. (BR) (UFVB); Martyniaceae: Martynia annua L. (CU) (Hernandez; Henry, 2010); Poaceae: Brachiaria decumbens Stapf (BR: GO, MG, ES, SP) (Ruiz, 2014); Rutaceae: Citrus spp. (BR: RS) (Coelho, 2008); Solanaceae: Nicotiana tabacum L. (CU; PR; BR: MG) (UFVB; Capriles, 1969; Ferreira et al., 2001; Hernandez; Henry, 2010; Van Lenteren et al., 2016), Solanum lycopersicum L. (CU) (Hernandez; Henry, 2010).
Macrolophus basicornis (Stal 1860): Fabaceae: Vigna unguiculata (L.) Walp. (BR: PI) (UFVB); Solanaceae: Nicotiana tabacum (L.) (BR: MG) (Van Lenteren et al., 2016).

Macrolophus praeclarus (Distant 1884): Amaranthaceae: Amaranthus spinosus (L.) (PR) (Capriles, 1969); Cucurbitaceae: Cucurbita sp. (NI) (Maes; Carvalho, 1989), Cucurbita moschata (Duchesne) Duchesne ex Poir. (PR) (Capriles, 1969); Euphorbiaceae: Jatropha gossypiifolia L. (PR) (Capriles, 1969); Martyniaceae: Martynia annua L. (CU) (Hernandez; Henry 2010); Poaceae: Panicum maximum cv. Mombaça Jacq. (BR: SP, GO, MG, SC, ES) (Ruiz, 2014); Solanaceae: Nicotiana tabacum L. (CU; NI; PR; BR: MG, GO) (Capriles, 1969; Alayo, 1974; Ferreira; Rossi, 1979; Ferreira et al., 2001; Hernandez; Henry, 2010; Maes; Carvalho, 1989), Solanum lycopersicum L. (BR: SP) (UFVB).

Monalonion annulipes V. Signoret, 1858: Lauraceae: Persea americana Mill. (BR) (UFVB); Malvaceae: Theobroma cacao L. (NI; CO; CR; EC; GT; GY; MX; PA; VE; BR: MG, AM) (Carvalho, 1972; Maes; Carvalho, 1989; Wheeler, 2001); Myrtaceae: Psidium guajava L. (BR: ES) (Boti et al., 2016); Rosaceae: Rubus sp. (FR) (Abreu, 1977); Urticaceae: Cecropia pachystachya Trec. (BR) (UFVB).

Monalonion atratum Distant, 1883: Malvaceae: Theobroma cacao L. (NI; BR: BA, GO, AM) (Silva et al., 1968; Carvalho, 1972; Maes; Carvalho, 1989); Rubiaceae: Coffea arabica L. (BR) (UFVB); 
Urticaceae: Cecropia pachystachya Trec. (BR: BA) (Silva et al., 1968).

Monalonion bahiense Costa Lima, 1938: Malvaceae: Theobroma cacao L. (BR: MG, BA) (Silva et al., 1968; CarvaIho, 1972; Ferreira et al., 2001); Rubiaceae: Hamelia patens Jacq. (BR) (CarvaIho, 1972).

Monalonion bondari Costa Lima, 1938: Malvaceae: Theobroma cacao L. (BR: BA) (Silva et al., 1968; Carvalho, 1972; Wheeler, 2001).

Monalonion dissimulatum Distant, 1883: Malvaceae: Theobroma cacao L. (NI; PE; BR: AM, BA) (Silva et al., 1968; Carvalho, 1972; Maes; Carvalho, 1989; Wheeler, 2001).

Monalonion itabunensis Carvalho, 1972: Malvaceae: Theobroma cacao L. (BR: BA, PA) (Carvalho, 1972).

Monalonion parviventre Herrich-Schaeffer, 1850: Malvaceae: Theobroma cacao L. (BR) (UFVB).

Monalonion schaefferi Stal, 1860: Anacardiaceae: Anacardium occidentale L. (Namyatova; Cassis, 2016); Begoniaceae: Begonia reniformis Dryand. (BR) (Silva et al., 1968), Begonia sp. (BR: BA) (Silva et al., 1968); Begonia spp. (Namyatova; Cassis, 2016); Malvaceae: Theobroma cacao L. (BR: BA) (Silva et al., 1968); Moraceae: Ficus sp. (Namyatova; Cassis, 2016); Rubiaceae: Hamelia patens Jacq. (Namyatova; Cassis, 2016); Urticaceae: Cecropia pachystachya Trec. (Namyatova; Cassis, 2016).
Tupiocoris agilis (Uhler, 1877): Geraniaceae: Geranium spp. (BR; CL; US) (Kelton, 1980a); Rosaceae: Rubus sp. (CA; US; UK) (Lindberg, 1958); Solanaceae: Physalis peruviana L. (BR) (UFVB).

Tupiocoris chlorogaster (Berg, 1878): Apiaceae: Petroselinum sativum Hoffm. (BR) (UFVB); Solanaceae: Petunia axillaris (Lam.) Britton et al. (BR: RJ, SC, SP) (Silva et al., 1968).

Tupiocoris cucurbitaceus (Spinola, 1852): Asteraceae: Urolepis hecatantha (DC.) R. King \& H. Rob. (AR; PY) (Logarzo et al., 2005); Cucurbitaceae (BR: MG, SP, PR, RJ) (Silva et al., 1968; Ferreira; Rossi, 1979); Geraniaceae: Geranium spp. (BR; CL; US) (Kelton, 1980a); Poaceae: Cynodon spp. (BR: SC, ES) (Ruiz, 2014); Rosaceae: Rubus sp. (CA) (Lindberg, 1958); Solanaceae: Nicotiana tabacum L. (BR: MG) (Ferreira; Rossi, 1979); Solanum lycopersicum L. (BR; AR) (UFVB; Ferreira et al., 2001; López et al., 2012).

Tupiocoris notatus (Distant, 1893): Solanaceae: Nicotiana tabacum L. (BR: MG, RS, AM, SP, BA, RJ) (Silva et al., 1968), Solanum lycopersicum L. (BR: MG, RS, AM, SP, BA, RJ) (Silva et al., 1968), Solanum tuberosum L. (BR: MG, RS, AM, SP, BA, RJ) (Silva et al., 1968).

\section{Eccritotarsini}

Aspidobothrys dimidiatus (Stal, 1860): Apocynaceae: Tabernaemontana hystrix Steud. (BR: MG) (Ferreira; Rossi, 1979), Thevetia sp. (BR: MG) (UFVB; Ferreira et al., 2001); Euphorbiaceae: 
Sapium haematospermum Müll.Arg. (BR: MG) (UFVB; Ferreira et al., 2001).

Aspidobothrys flavicosta (Carvalho, 1949): Euphorbiaceae: Sapium haematospermum Müll.Arg. (BR: MG, GO) (UFVB; Silva et al., 1968).

Aspidobothrys ruficeps (Berg, 1878): Solanaceae: Solanum tuberosum L. (BR: RS) (Silva et al., 1968).

Bothrophorella nigra (Stal, 1860): Convolvulaceae: Ipomoea batatas (L.) Lam. (BR: MG) (Silva et al., 1968; Ferreira; Rossi, 1979; Ferreira et al., 2001).

Cyrtocapsus caligineus (Stal, 1859): Asteraceae (CU) (Hernandez; Henry, 2010); Bromeliaceae: Ananas comosus (L.) Merr. (CU) (Hernandez; Henry, 2010); Convolvulaceae: Ipomoea batatas (L.) Lam. (CU; PR) (Capriles, 1969; Hernandez; Henry, 2010); Ipomoea purpurea (L.) Roth (US) (Wheeler, 2001); Ipomoea sp. (CU) (Hernandez; Henry, 2010), Jacquemontia nodiflora (Desr.) G. Don. (CU) (Hernandez; Henry, 2010), Turbina corymbosa (L.) Raf. (CU) (Hernandez; Henry, 2010), Cucurbitaceae: Cucurbita maxima Duch. Lam. (CU) (Hernandez; Henry, 2010), Cucurbita moschata (Duchesne) Duchesne ex Poir. (PR) (Capriles, 1969), Cucurbita sp. (CU) (Alayo, 1974); Cucurbitaceae (NI) (Maes; CarvaIho, 1989).

Cyrtocapsus femoralis Reuter, 1892: Convolvulaceae: Ipomoea batatas (L.) Lam. (BR: MG) (Ferreira; Rossi, 1979); Fabaceae: Vigna unguiculata (L.) Walp. (BR: MG, RS, PI) (UFVB); Poaceae: As- sociação entre Brachiaria brizantha (A. Rich.) Stapf. e Panicum maximum cv. Mombaça Jacq. (BR: ES) (Ruiz, 2014).

Eccritotarsus catarinenses (CarvaIho, 1948): Pontederiaceae: Eichhornia crassipes (Mart.) Solms (Wheeler, 2001).

Eurychilella cinnabarina Carvalho, 1953: Bromeliaceae: Billbergia sp. (BR) (Carvalho, 1953; Silva et al., 1968).

Eurychilella discoidalis (Reuter, 1912): Araceae: Caladium bicolor (Aiton) Vent. (BR: MG, RJ) (Silva et al., 1968), Caladium sp. (BR: MG) (Ferreira; Rossi, 1979; Ferreira et al., 2001).

Eurychilella figueiredoi (Carvalho, 1944): Bromeliaceae: Aechmea nudicaulis (L.) Griseb. (BR: RJ) (Silva et al., 1968).

Eurychilella nigra Carvalho e Rosas, 1962: Piperaceae: Piper aduncum L. (BR) (UFVB); Rutaceae: Pilocarpus jaborandi Holmes (BR: RJ) (Silva et al., 1968).

Eurychiloides bilobosus Carvalho e Gomes, 1971: Piperaceae: Piper gaudichaudianum Kunth (BR) (UFVB).

Mecolaemus carvalhoi (Costa Lima, 1942): Orchidaceae: Cattleya sp. (BR: PE) (Silva et al., 1968).

Mecolaemus fasciatus Hsiao, 1947: Orchidaceae: Oncidium sp. (BR) (Hsiao, 1947).

Neella caipora Carvalho, 1948: Araceae: Philodendron sp. (BR: MG) (Carvalho, 1948). 
Neella carmelitana Carvalho, 1945: Lamiaceae: Leonotis nepetifolia (L.) R.Br. (BR: MG) (Silva et al., 1968; Ferreira et al., 2001).

Neella lutescens (Stal, 1860): Araceae: Philodendron propinquum Schott (BR: RJ) (UFVB).

Neella mantiqueirae Carvalho, 1954: Orchidaceae: Epidendrum sp. (BR) (UFVB).

Neella similaris Carvalho e Gomes, 1971: Araceae (BR) (UFVB).

Neoneella bosqi Carvalho, 1946: Araceae: Philodendron sp. (AR) (Carvalho, 1946; Wheeler, 2001).

Neoneella milzae Carvalho, 1946: Araceae: Philodendron sp. (BR: RJ) (Silva et al., 1968).

Neoneella minúscula Carvalho, 1985: Araceae: Philodendron sp. (BR: MG) (Ferreira et al., 2001); Orchidaceae (BR: MG) (Ferreira et al., 2001).

Neoneella paranaensis Carvalho, 1946: Araceae (BR: RJ) (UFVB).

Neoneella zikani Costa Lima, 1942: Araceae: Philodendron sp. (BR: RJ) (Silva et al., 1968); Orchidaceae: Cattleya perrinii Lindl. (BR: RJ) (Silva et al., 1968).

Pachymeroceroides bromeliae (Carvalho, 1946): Bromeliaceae: Aechmea sp. (BR: RJ) (Silva et al., 1968; Uceli et al., 2009).

Pachymerocerus fairmairei (Stal, 1860): Balsaminaceae: Impatiens walleriana Hook. Fil. (BR: SC) (Silva et al., 1968).
Parafurius discifer (Stal, 1860): Araceae: Zantedeschia aethiopica (L.) Spreng. (BR:MG) (UFVB); Rubiaceae: Coffea Arabica L. (BR: MG) (Ferreira et al., 2001), Coffea sp. (BR: MG) (Silva et al., 1968; Ferreira; Rossi, 1979).

Pycnoderes atratus (Distant, 1884): Fabaceae: Phaseolus vulgaris L. (NI) (Maes; Carvalho, 1989).

Pycnoderes incurvus (Distant, 1884): Asteraceae: Calendula officinalis L. (BR: MG) (UFVB); Cucurbitaceae: Lagenaria siceraria (Molina) Standl. (BR: MG, RJ) (Silva et al., 1968; Ferreira; Rossi, 1979; Ferreira et al., 2001), Sechium edule Sw. (BR: MG, RJ) (Silva et al., 1968; Ferreira; Rossi, 1979; Ferreira et al., 2001).

Pycnoderes palustris Carvalho, 1951: Alismataceae: Echinodorus macrophyllus (Kunth) Micheli (BR) (UFVB).

Pycnoderes quadrimaculatus Guerin-Meneville, 1857: Amaranthaceae: Amaranthus sp. (NI; BR: MG) (Maes; Carvalho, 1989; Ferreira et al., 2001); Araceae: Zantedeschia aethiopica (L.) Spreng. (BR) (UFVB); Convolvulaceae: Ipomoea sp. (CU) (Hernandez; Henry, 2010); Cucurbitaceae: Cucumis sativus L. (CU) (Hernandez; Henry, 2010), Cucumis sp. (NI) (Maes; Carvalho, 1989), Cucurbita máxima Duch. Lam. (NI; BR: MG) (Ferreira et al., 2001; Maes; CarvaIho, 1989), Cucurbita pepo L. (CU) (Hernandez; Henry, 2010); Fabaceae: Vigna unguiculata (L.) Walp. (BR: PI) (UFVB), Phaseolus vulgaris (A. Rich.) (NI) (Maes; Carvalho, 1989); Poaceae: Brachiaria 
brizantha (A. Rich.) Stapf (BR: ES) (Ruiz, 2014).

Pycnoderes sixeonotoides Carvalho e Hussey, 1954: Commelinaceae: Commelina virginica $\mathrm{L}$. (BR) (UFVB).

Sinervus baerensprungi Stal, 1860: Heliconiaceae: Heliconia psittacorum L.f. (BR: MG, RJ) (Carvalho, 1945; Silva et al., 1968; Ferreira; Rossi, 1979; Ferreira et al., 2001).

Sixeonotus brasiliensis Carvalho e Gomes, 1971: Convolvulaceae: Ipomoea batatas (L.) Lam. (BR: MG) (Ferreira et al., 2001); Fabaceae: Phaseolus vulgaris L. (BR: MG) (UFVB).

Spartacus albatus Distant, 1884: Heliconiaceae: Heliconia psittacorum L.f. (BR: MG) (Carvalho, 1945; Silva et al., 1968; Ferreira; Rossi, 1979; Ferreira et al., 2001).

Spartacus discovittatus Carvalho, 1945: Costaceae: Costus spiralis (Jacq.) Roscoe (BR: MG) (Carvalho, 1945; Silva et al., 1968; Ferreira; Rossi, 1979); Heliconiaceae: Heliconia psittacorum L.f. (BR: MG) (Carvalho, 1945; Silva et al., 1968; Ferreira; Rossi, 1979; Ferreira et al., 2001).

Spartacus minensis Carvalho, 1985: Heliconiaceae: Heliconia psittacorum L.f. (BR: MG) (Ferreira et al., 2001).

Spartacus tenuis Carvalho, 1945: Marantaceae: Thalia dealbata Fraser (BR: MG, RJ) (Carvalho, 1945; Silva et al., 1968).

Sysinas centralis Distant, 1883: Euphorbiaceae: Sapium sp. (BR: MG) (Silva et al., 1968; Ferreira et al., 2007; Hernandez; Henry, 2010).

Sysinas pallidipes (Stal, 1860): Euphorbiaceae: Sapium sp. (BR: MG) (UFVB; Ferreira; Rossi, 1979).

Tenthecoris bicolor J. Scott, 1886: Orchidaceae: Acineta sp. (BR) (UFVB), Lycaste sp. (GT) (Hsiao; Sailer, 1947), Orchidaceae (GT) (Hsiao; Sailer, 1947), Rhynchostele bictoniensis Soto Arenas \& Salazar (GT) (Hsiao; Sailer, 1947), Rossioglossum grande (Lindl.) Garay \& G. C.Kenn. (GT) (Hsiao; Sailer, 1947).

Tenthecoris figueiredoi Carvalho, 1950: Fabaceae: Newtonia sp. (BR: SP) (Carvalho, 1950); Orchidaceae: Dendrobium sp. (BR: SP) (UFVB; Silva et al., 1968), Gomesa planifolia (Lindl.) Klotzsch ex Rchb.f. (BR: SP) (Carvalho, 1950), Miltonia regnellii Rchb. F. (BR: SP) (Carvalho, 1950), Oncidium sp. (BR: SP) (Carvalho, 1950; Silva et al., 1968).

Tenthecoris nanus Carvalho, 1948: Araceae: Caladium spp. (BR: MG) (Ferreira et al., 2001).

Tenthecoris orchidearum (Reuter, 1902): Amaryllidaceae: Hippeastrum sp. (BR: SP) (UFVB); Orchidaceae: Cattleya aclandiae Lindl. (BR: MG) (Ferreira; Rossi, 1979; Ferreira et al., 2001), Cattleya labiate Lindl. (BR: MG) (Ferreira; Rossi, 1979; Ferreira et al., 2001), Cattleya perrinii Lindl. (BR: MG) (Ferreira; Rossi, 1979, Ferreira et al., 2001), Cattleya spp. (BR: MG) (UFVB).

Zikaniola elegans Carvalho, 1946: Araceae: Philodendron sp. (BR: RJ) (Carvalho, 1946; Silva et al., 1968). 


\section{Deraeocorinae}

\section{Clivinematini}

Ambracius dufouri Stal, 1860: Onagraceae: Fuchsia regia (Vand. ex Vell.) Munz (BR: MG) (Ferreira et al., 2001).

Ofellus mantiqueiranus Carvalho e Sailer, 1953: Asteraceae: Ageratina adenophora (Spreng.) R. King \& H. Rob. (BR: MG) (Ferreira et al., 2001); Verbenaceae: Lantana câmara L. (BR: MG) (Ferreira et al., 2001).

\section{Deraeocorini}

Lundiella reinhardti Carvalho, 1951: Araceae (BR) (UFVB).

\section{Hyaliodini}

Annona bimaculata (Distant, 1884): Convolvulaceae: Ipomoea batatas (L.) Lam. (BR: MG) (Silva et al., 1968; Ferreira et al., 2001); Solanaceae: Solanum lycopersicum L. (BR) (UFVB).

Brasiliocarnus incaicus Carvalho, 1985: Bixaceae: Bixa orellana (BR: MG) (UFVB).

Carijoanus ruberfasciatus (Carvalho, 1945): Monimiaceae (BR: MG) (CarvaIho, 1945; Silva et al., 1968); Urticaceae: Cecropia sp. (BR: MG) (Carvalho, 1945; Ferreira et al., 2001).

Hyaliodes beckeri Carvalho, 1953: Euphorbiaceae: Manihot esculenta Crantz (BR: DF) (Oliveira et al., 2002).

Hyaliodes glabratus (Distant, 1888): Dennstaedtiaceae: Pteridium caudatum (L.) Maxon (UY) (Carvalho; Drake, 1943); Euphorbiaceae: Manihot escu- lenta Crantz (UY; BR: MG) (Carvalho; Drake, 1943; Carvalho, 1945; Ferreira et al., 2001); Rosaceae: Rubus sp. (UY) (Carvalho; Drake, 1943).

Hyaliodes vitreus (Distant, 1884): Cucurbitaceae: Cayaponia diversifolia (Cogn.) Cogn. (BR) (UFVB); Euphorbiaceae: Manihot esculenta Crantz (BR: MG) (Ferreira; Rossi, 1979; Ferreira et al., 2001).

Hyaliodocoris clarus (Stal, 1860): Cucurbitaceae: Cayaponia diversifolia (Cogn.) Cogn. (BR: MG) (Carvalho, 1945; Silva et al., 1968; Ferreira; Rossi, 1979; Ferreira et al., 2001).

Hyaliodocoris insignis (Stal, 1860): Asteraceae: Conyza spp. (BR) (UFVB), Gamochaeta falcata (Lam.) Cabrera (BR) (UFVB); Fabaceae: Glycine max (L.) Merr. var. bilox (BR) (UFVB), Phaseolus vulgaris L. (BR: MG) (Ferreira et al., 2001); Rosaceae: Fragaria $x$ ananassa Duch. (BR: PR) (Zawadneak et al., 2016); Solanaceae: Solanum lycopersicum L. (BR) (UFVB).

Paracarnus myersi China, 1931: Piperaceae: Piper peltatum L. (CU) (Alayo, 1974; Hernandez; Henry, 2010).

Perissobasis heroni Ferreira e CoeIho, 2009: Rubiaceae: Coffea Arabica L. (BR: MG) (UFVB; Ferreira et al., 2009).

\section{Termatophylini}

Termatophylidea opaca Carvalho, 1955: Malvaceae: Theobroma cacao L. (GY) (Ferreira, 1993); Rosaceae: Eriobotrya japonica (Thunb.) Lindl. (BR: MG) (UFVB; Ferreira, 1993; Ferreira et al., 
2001); Rubiaceae: Nauclea latifolia Sm. (BR: MG) (Ferreira, 1993; Ferreira et al., 2001).

\section{Cylapinae}

\section{Fulviini}

Fulvius anthocoroides Stal, 1862: Araceae: Aglaonema sp. (América) (Carvalho; Costa, 1994); Cycadaceae (América) (Carvalho; Costa, 1994); Fabaceae: Erythtrina sp. (NI) (Maes; Carvalho, 1989), Phaseolus vulgaris L. (América) (Carvalho; Costa, 1994); Meliaceae: Swietenia sp. (NI) (Maes; Carvalho, 1989), Swietenia macrophyla King (América) (Carvalho; Costa, 1994); Moraceae: Morus sp. (América) (Carvalho; Costa, 1994); Passifloraceae: Passiflora edulis Sims (América) (Carvalho; Costa, 1994); Poaceae: Zea mays L. (América) (CarvaIho; Costa, 1994) Rutaceae: Citrus paradisi Macfad. (América) (Carvalho; Costa, 1994); Solanaceae: Solanum tuberosum L. (América) (Carvalho; Costa, 1994).

Fulvius bisbistillatus (Stal, 1860): Convolvulaceae: Ipomoea sp. (BR: MG) (Ferreira et al., 2001).

Fulvius breddini Reuter, 1902: Orchidaceae (BR: MG) (Ferreira et al., 2001).

Fulvius ornatifrons Carvalho, 1955: Orchidaceae: Cattleya lueddemanniana Rchb.f. (América) (Carvalho, 1955), Cattleya sp. (América; VE) (Carvalho, 1955;
Carvalho; Costa, 1994), Orchidaceae (América; VE) (Carvalho, 1955; CarvaIho; Costa, 1994).

Fulvius quadristillatus (Stal, 1860): Orchidaceae: Laelia sp. (América; NI) (Carvalho; Costa, 1994; Maes; Carvalho, 1989), Odontoglossum sp. (NI) (Maes; Carvalho, 1989), Rossioglossum grande (Lindl.) Garay \& G.C.Kenn (América) (Carvalho; Costa, 1994), Orchidaceae (América) (Carvalho; Costa, 1994).

Peritropsis saldaeformis Uhler, 1891: Juglandaceae: Carya sp. (GE; NI) (Henry; Smith, 1979; Maes; Carvalho, 1989).

\section{Orthotylinae}

Halticini

Microtechnites bractatus (Say, 1832): Amaranthaceae: Amaranthus sp. (NI) (Maes; Carvalho, 1989); Apiaceae: Apium sp. (NI) (Maes; Carvalho, 1989), Carum petroselinum (L.) (BR: MG) (Ferreira; Rossi, 1979), Petroselinum crispum (Mill.) (BR: MG, SP) (Silva et al., 1968; Ferreira et al., 2001); Asteraceae: Cirsium sp. (NI) (Maes; Carvalho, 1989), Lactuca sativa L. (NI) (Maes; Carvalho, 1989); Brassicaceae: Brassica napus L. (BR: MG) (Ferreira et al., 2001), Brassica oleracea (NI) (Maes; Carvalho, 1989), Brassica rapa subs. Rapa L. (BR: MG, SP) (Silva et al., 1968; Ferreira et al., 2001), Brassica sp. (BR: MG) (Silva et al., 1968; Ferreira et al., 2001), Raphanus raphanistrum L. (BR: SP) (Silva et al., 1968), Raphanus sativus L. (NI) 
(Maes; Carvalho, 1989); Convolvulaceae: Ipomoea batatas (L.) Lam. (NI) (Maes; Carvalho, 1989; Tatarnic; Cassis, 2012); Cucurbitaceae: Bryonia sp. (NI) (Maes; Carvalho, 1989), Citrullus lanatus (Thunb.) Matsumura \& Nakai (NI) (Maes; Carvalho, 1989), Cucumis sp. (NI) (Maes; Carvalho, 1989), Cucurbita moschata (Duchesne) Duchesne ex Poir. (PR) (Capriles, 1969), Lagenaria siceraria (Molina) Standl. (NI) (Maes; Carvalho, 1989), Sechium edule Sw. (NI) (Maes; Carvalho, 1989); Fabaceae: Glycine max (L.) Merr. (BR) (UFVB), Medicago sativa L. (US) (Day; Saunders, 1990), Medicago sp. (NI) (Maes; Carvalho, 1989), Phaseolus vulgaris L. (CU) (Alayo, 1974), Trifolium sp. (NI) (Maes; Carvalho, 1989), Vigna unguiculata (L.) Walp. (NI) (Maes; CarvaIho, 1989); Fagaceae: Quercus sp. (GE) (Henry; Smith, 1979); Juglandaceae: Juglans sp. (NI) (Maes; Carvalho, 1989); Malvaceae: Gossypium hirsutum L. (NI) (Maes; Carvalho, 1989); Oleaceae: Fraxinus sp. (NI) (Maes; Carvalho, 1989; Tatarnic; Cassis, 2012); Pedaliaceae: Sesamum indicum L. (NI) (Maes; CarvaIho, 1989); Phytolaccaceae: Phytolacca sp. (NI) (Maes; Carvalho, 1989); Poaceae: Avena sp. (NI) (Maes; Carvalho, 1989), Hordeum sp. (NI) (Maes; CarvaIho, 1989), Sorghum bicolor (L.) Moench. (NI) (Maes; Carvalho, 1989), Triticum sp. (NI) (Maes; Carvalho, 1989), Zea mays L. (NI) (Maes; Carvalho, 1989); Portulacaceae: Portulaca sp. (NI) (Maes; Carvalho, 1989); Rutaceae: Citrus sp. (NI) (Maes; Carvalho, 1989); Solanaceae: Capsicum annuum L. (NI) (Maes; Car- valho, 1989), Nicotiana tabacum L. (NI) (Maes; Carvalho, 1989), Solanum lycopersicum L. (BR; CU; NI) (Maes; Carvalho, 1989; Hernandez; Henry, 2010; Wheeler, 2001), Solanum sp. (NI) (Maes; Carvalho, 1989).

Microtechnites spegazzini (Berg, 1883): Asteraceae: Picris helminthioides (Ball) Greuter (BR) (UFVB); Brassicaceae: Brassica sp. (BR) (UFVB).

\section{Orthotylini}

Adfalconia cunealis Carvalho e Rosas, 1962: Euphorbiaceae: Acalypha brasiliensis Müll.Arg. (BR: MG, SC, RJ) (Carvalho; Rosas, 1962; Silva et al., 1968; Ferreira; Rossi, 1979; Ferreira et al., 2001).

Adhyalochloria inermis (Carvalho, 1985): Fuchsia regia (Vand. ex Vell.) (BR) (UFVB).

Brasiliomiris ernestoi Carvalho, 1946: Urticaceae: Cecropia sp. (BR: MG) (Silva et al., 1968; Ferreira; Rossi, 1979; Ferreira et al., 2001).

Ceratocapsus pilosulus Knight, 1930: Fagaceae: Quercus microphylla Née (CA) (Kelton, 1980b; Wheeler, 2001), Quercus pubescens Willd. (CA) (Kelton, 1980b; Wheeler, 2001).

Itacoris nigrioculis Carvalho, 1947: Boraginaceae: Cordia laevigata Lam. (PR) (Capriles, 1969); Piperaceae: Piper sp. (PR) (Capriles, 1969).

Jobertus esavianus Carvalho, 1944: Cucurbitaceae: Cucurbita moschata (Duchesne) Duchesne ex Poir. (BR: MG) 
(Ferreira et al., 2001); Fabaceae: Phaseolus vulgaris L. (BR: MG) (Ferreira et al., 2001); Lamiaceae: Clerodendrum chinense (Osbeck) Mabb. (BR: MG) (Ferreira et al., 2001), Clerodendrum sp. (BR: MG) (Ferreira et al., 2001); Solanaceae: Solanum cernuum Vell. (BR: MG) (Carvalho, 1944b).

Papaveronia bergi Carvalho e Carpintero, 1992: Solanaceae: Solanum donianum Walp. (BR) (UFVB).

Saileria almeidai (Carvalho, 1946): Solanaceae (BR: MG, SC) (Silva et al., 1968; Ferreira et al., 2001).

Saileria sulina Carvalho, 1989: Euphorbiaceae: Alchornea sidifolia Müll. Arg. (BR: SC) (Carvalho, 1989).

Sericophanes niger Poppius, 1921: O gênero Sericophanes é frequentemente encontrado em Poaceae (Carvalho, 1944a).

Sericophanes obscuricornis Poppius, 1921: O gênero Sericophanes é frequentemente encontrado em Poaceae (Carvalho, 1944a).

Sericophanes ornatus (Berg, 1878): Fabaceae: Medicago sativa L. (BR: MG) (Ferreira et al., 2001); Associação entre Poaceae: Brachiaria mutica (Forssk.) Stapf e Brachiaria ruziziensis R.Germ. \& C.M.Evrard (BR: ES) (Ruiz, 2014).

Sericophanes scotti (Berg, 1883): O gênero Sericophanes é frequentemente encontrado em Poaceae (Carvalho, 1944a).

Solanocoris semiruber Carvalho, 1945: Solanaceae: Solanum bullatum
Vell. (BR: MG) (Ferreira et al., 2001), Solanum cernuum Vell. (BR: MG) (Ferreira et al., 2001), Solanum donianum Walp. (BR: MG) (Ferreira et al., 2001).

\section{Mirinae}

Mirini

Creontiades pallidus (Rambur, 1839): Fabaceae: Arachis pintoi Krapov. \& W.C. Greg. (BR: MT) (UFVB); Indigofera tinctoria L. (CA; US; UK) (UFVB; Lindberg, 1958).

Creontiades purgatus (Stal, 1860): Apiaceae: Daucus carota L. (Ferreira et al., 2001); Associação entre Poaceae Sorghum bicolor (L.) Moench e Brachiaria ruziziensis R.Germ. \& C.M.Evrard (BR: MT) (UFVB). Associação entre Fabaceae: Cajanus cajan (L.) Millsp. e Poaceae: Eleusine coracana (L.) Gaertn. (BR: MT) (UFVB); Convolvulaceae: Ipomoea sp. (BR: MG) (Ferreira et al., 2001); Euphorbiaceae: Manihot esculenta Crantz (BR) (Silvie; Thomazoni, 2007); Fabaceae: Arachis pintoi Krapov. \& W.C. Greg (BR: MT) (UFVB; Silvie; Thomazoni, 2007), Crotalaria juncea L. (BR: MT) (UFVB; Silvie; Thomazoni, 2007), Glycine max (L.) Merr. (BR) (Silvie; Thomazoni, 2007); Pedaliaceae: Sesamum sp. (BR: MT) (Silvie; Thomazoni, 2007); Poaceae: Brachiaria ruziziensis R.Germ. \& C.M.Evrard (BR: MT) (Silvie; Thomazoni, 2007), Cynodon dactylon (L.) Pers. (BR: MT) (Silvie; Thomazoni, 2007), Eleusine coracana (L.) Gaertn. (BR: MT) (Silvie; Thomazoni, 2007), Zea mays L. (BR: 
MG) (Ferreira et al., 2001; Silvie; Thomazoni, 2007).

Creontiades rubrinervis (Stal, 1862): Convolvulaceae: Ipomoea pes-caprae (L.) R.Br. (CU) (Hernandez; Henry, 2010), Ipomoea sp. (BR: MG; CU) (Ferreira et al., 2001; Hernandez; Henry, 2010); Cucurbitaceae: Citrullus lanatus (Thunb.) Matsumura \& Nakai (NI) (Maes; Carvalho, 1989), Cucumis sp. (NI) (Maes; Carvalho, 1989); Fabaceae: Canavalia rosea (Sw.) DC. (CU) (Hernandez; Henry, 2010), Canavalia sp. (BR: MG; CU) (Ferreira et al., 2001; Hernandez; Henry, 2010), Cicer arietinum L. (CU) (Hernandez; Henry, 2010); Glycine max (NI) (Maes; Carvalho, 1989), Phaseolus lunatus (CU) (Alayo, 1974; Hernandez; Henry, 2010), Phaseolus sp. (BR: MG) (Ferreira et al., 2001), Phaseolus vulgaris L. (BR: MG; CU) (Alayo, 1974; Maes; CarvaIho, 1989; Ferreira et al., 2001; Hernandez; Henry, 2010), Vigna sp. (NI) (Maes; Carvalho, 1989), Vigna unguiculata (L.) Walp. (BR: MG, PI; CU) (Alayo, 1974; Ferreira; Rossi, 1979; Maes; Carvalho, 1989; Ferreira et al., 2001; Hernandez; Henry, 2010), Malvaceae: Gossypium hirsutum L. (BR: SP, MG; NI) (Silva et al., 1968; Maes; Carvalho, 1989; Ferreira et al., 2001; Wheeler, 2001), Gossypium herbaceum L. (BR: MG) (Ferreira; Rossi, 1979); Pedaliaceae: Sesamum indicum L. (NI) (Maes; Carvalho, 1989); Poaceae: Sorghum bicolor (L.) Moench (NI) (Maes; Carvalho, 1989), Zea mays L. (NI) (Maes; Carvalho, 1989); Solanaceae: Solanum sp. (NI) (Maes; Carvalho, 1989).
Dagbertus amapaensis Carvalho, 1988: Melastomataceae: Clidemia hirta (L.) D.Don (BR) (UFVB), Pterolepis glomerata (Rottb.) Miq. (BR) (UFVB).

Dagbertus minensis Carvalho e Fontes, 1983: Lauraceae: Persea americana Mill. (BR) (UFVB).

Dagbertus phaleratus (Berg, 1892): Asteraceae: Mikania cordifolia (L. Fil.) Willd. (BR) (UFVB); Lamiaceae: Condea undulata (Schrank) Harley \& J.F.B. Pastore (BR) (UFVB).

Derophthalma minuscula Carvalho, 1944: Asteraceae: Anthemis cotula L. (BR: MG) (Ferreira; Rossi, 1979); Euphorbiaceae: Acalypha brasiliensis Müll. Arg. (BR: MG) (Ferreira; Rossi, 1979).

Derophthalma reuteri Berg, 1883: Asteraceae: Anthemis cotula L. (BR: MG) (Ferreira; Rossi, 1979).

Garganus gracilentus (Stal, 1860): Amaranthaceae: Beta vulgaris L. (BR: MG) (Ferreira et al., 2001); Apiaceae: Daucus carota L.(BR: MG) (Ferreira et al., 2001); Aquifoliaceae: Ilex paraguariensis A. St.-Hil. (BR: MG) (UFVB); Associação entre Fabaceae: Cajanus cajan (L.) Millsp. e Poaceae: Eleusine coracana (L.) Gaertn. (BR: MT) (UFVB); Associação entre Poaceae Panicum maximum Jacq. e Brachiaria brizantha (A. Rich.) Stapf (BR: ES) (Ruiz, 2014); Associação entre Poaceae: Brachiaria ruziziensis R.Germ. \& C.M.Evrard e Sorghum bicolor (L.) Moench (BR: MT) (UFVB); Asteraceae: Bidens pilosa L. (BR: MG) (Ferreira et al., 2001; Ferreira; 
Rossi, 1979), Bidens rubicundula f. alba T.G.J. Rayner (BR: MG) (Ferreira; Rossi, 1979), Calendula officinalis L. (BR: MG) (UFVB); Brassicaceae: Brassica sp. (NI) (Maes; Carvalho, 1989), Raphanus raphanistrum L. (BR: MG) (Ferreira; Rossi, 1979); Convolvulaceae: Ipomoea batatas (L.) Lam. (BR: MG) (Silva et al., 1968; Ferreira; Rossi, 1979; Ferreira et al., 2001), Ipomoea sp. (BR: MG) (Ferreira et al., 2001), Solanum tuberosum L. (BR: MG) (Silva et al., 1968; Ferreira; Rossi, 1979); Fabaceae: Glycine max (L.) Merr. (BR: MG) (Silva et al., 1968; Ferreira; Rossi, 1979; Ferreira et al., 2001), Phaseolus vulgaris L. (BR: MG) (Silva et al., 1968; Ferreira; Rossi, 1979; Ferreira et al., 2001); Malvaceae: Abelmoschus esculentus (L.) Moench (BR: MG) (Silva et al., 1968; Ferreira; Rossi, 1979; Ferreira et al., 2001), Gossypium herbaceum L. (BR: MG) (Ferreira; Rossi, 1979), Gossypium hirsutum L. (BR: MG) (Silva et al., 1968; Ferreira et al., 2001; Silvie; Thomazoni, 2007), Sida sp. (BR: MG) (Silva et al., 1968; Ferreira; Rossi, 1979; Ferreira et al., 2001); Poaceae: Triticum sp. (Ferreira et al., 2001), Oryza sativa L. (BR) (Coelho, 2008).

Henicocnemis amazonica Carvalho e Costa, 1993: Apiaceae: Petroselinum sp. (BR) (UFVB).

Horcias guapeanus Carvalho, 1976: Convolvulaceae: Ipomoea sp. (BR: MG) (Ferreira et al., 2001); Fabaceae: Vigna unguiculata (L.) Walp. (BR: PI) (UFVB); Malvaceae: Gossypium hirsutum L. (BR: MG) (Ferreira et al., 2001).
Horcias pentheri Reuter, 1907: Malvaceae: Sida sp. (BR: PE) (Carvalho, 1975b); Rubiaceae: Borreria sp. (BR: PE) (Carvalho, 1975b).

Horciasinus signoreti Reuter, 1907: Amaranthaceae: Beta vulgaris L. (BR: MG) (Ferreira et al., 2001); Apiaceae: Daucus carota L. (BR: MG) (Ferreira et al., 2001); Associação entre Poaceae: Sorghum bicolor (L.) Moench and Brachiaria ruziziensis R.Germ. \& C.M.Evrard (BR: MT) (UFVB); Asteraceae: Sonchus sp. (BR: MG) (Ferreira; Rossi, 1979), Tagetes erecta L. (BR: MG) (UFVB); Brassicaceae: Raphanus raphanistrum L. (BR: MG) (Ferreira; Rossi, 1979); Fabaceae: Crotalaria juncea L. (BR: MT) (UFVB), Desmodium adscendens (Sw.) DC. (BR) (UFVB), Glycine max (L.) Merr. (BR: MT) (UFVB), Phaseolus vulgaris L. (BR: MG) (Ferreira et al., 2001), Vigna unguiculata (L.) Walp. (BR) (UFVB); Malvaceae: Gossypium hirsutum L. (BR: MG, MT) (Ferreira et al., 2001; Silvie; Thomazoni, 2007); Melastomataceae: Pterolepis glomerata (Rottb.) Miq. (BR) (UFVB), Pleroma trichopodum DC. (BR) (UFVB); Poaceae: Cynodon spp. (BR: ES) (Ruiz 2014), Digitaria insularis (L.) Mez ex Ekman (BR: MG) (UFVB), Oryza sativa L. (BR: MG) (UFVB).

Horciasisca insignis Carvalho, 1976: Fabaceae: Phaseolus vulgaris L. (BR: MG) (Ferreira et al., 2001); Poaceae: Zea mays L. (BR: MG) (Ferreira et al., 2001); Rubiaceae: Coffea Arabica L. (BR: MG) (Ferreira et al., 2001). 
Horciasoides nobilellus (Berg, 1883): Amaranthaceae: Amaranthus spinosus L. (BR: MG) (Ferreira et al., 2001; Ferreira; Rossi, 1979), Amaranthus viridis L. (BR) (Silvie; Thomazoni, 2007); Asteraceae: Baccharis dracunculifolia DC. (AR; PY) (Logarzo et al., 2005), Baccharis punctulate DC. (AR; PY) (Logarzo et al., 2005), Bidens pilosa L. (BR: MG) (Ferreira; Rossi, 1979; Ferreira et al., 2001), Bidens rubicundula $f$. alba T.G.J. Rayner (BR) (Silvie; Thomazoni, 2007), Eupatorium inulaefolium Kunt. (AR; PY) (Logarzo et al., 2005), Mikania cordifolia (L. Fil.) Willd. (AR; PY) (Logarzo et al., 2005), Solidago chilensis Meyen (AR; PY) (Logarzo et al., 2005); Hypericaceae: Hypericum sp. (AR; PY) (Logarzo et al., 2005); Lamiaceae: Condea undulata (Schrank) Harley \& J.F.B.Pastore (AR; PY) (Logarzo et al., 2005); Malvaceae: Abelmoschus esculentus (L.) Moench (BR: PR) (Silvie; Thomazoni, 2007), Gossypium herbaceum (L.) (BR) (UFVB), Sida sp. (BR: PR) (Silvie; Thomazoni, 2007), Gossypium hirsutum L. (BR: MG) (Silvie; Thomazoni, 2007; Ferreira et al., 2001; Wheeler, 2001), Hibiscus esculentus L. (BR: MG) (Ferreira et al., 2001), Malvastrum coromandelianum (L.) Garcke (BR: MG) (Ferreira; Rossi, 1979), Sida cordifolia L. (BR: MG) (Ferreira; Rossi, 1979; Ferreira et al., 2001), Sida rhombifolia L. (BR: MG) (Ferreira; Rossi, 1979; Ferreira et al., 2001), Triumfetta semitriloba Jacq. (BR: MG) (Ferreira; Rossi, 1979; Ferreira et al., 2001), Melastomataceae: Pterolepis glomerata (Rottb.) Miq. (BR) (UFVB), Pleroma trichopodum DC. (BR) (UFVB);
Rosaceae: Malus pumila Mill. (AR; PY) (Logarzo et al., 2005).

Lampethusa collaris Reuter, 1909: Verbenaceae: Lantana sp. (Wheeler, 2001).

Phytocoris aspersus Carvalho e Gomes, 1970: Asteraceae: Baccharis coridifolia DC. (BR) (UFVB), Baccharis dracunculifolia DC. (BR) (UFVB), Baccharis medulosa DC. (BR) (UFVB), Baccharis sp. (AR; PY) (Logarzo et al., 2005), Senecio grisebachii Baker (BR) (UFVB).

Phytocoris effictus Stal, 1860: Asteraceae: Parthenium hysterophorus L. (BR) (UFVB); Lamiaceae: Condea undulata (Schrank) Harley \& J.F.B. Pastore (BR) (UFVB).

Phytocoris subvittatus (Stal, 1860): Asteraceae: Baccharis dracunculifolia DC. (AR; PY) (Logarzo et al., 2005), Baccharis trimera (Less.) DC. (AR; PY) (Logarzo et al., 2005), Mikania cordifolia (L. Fil.) Willd. (AR; PY) (Logarzo et al., 2005), Parthenium hysterophorus L. (AR; PY) (Logarzo et al., 2005); Fabaceae: Mimosa arenosa (Willd.) Poir (Carvalho, 1975b), Mimosa sp. (BR: MG) (Ferreira et al., 2001), Phaseolus vulgaris L. (BR: MG) (Ferreira et al., 2001); Lamiaceae: Condea undulata (Schrank) Harley \& J.F.B.Pastore (AR; PY) (Logarzo et al., 2005).

Piasus cribricollis (Stal, 1860): Lauraceae: Persea americana Mill. (BR: MG, MT, RJ, SC) (Silva et al., 1968; Ferreira et al., 2001). 
Polymerus modestus (Blanchard, 1852): Apiaceae: Eryngium agavifolium Griseb. (BR) (UFVB).

Polymerus testaceipes (Stal, 1860): Amaranthaceae: Amaranthus dubius Mart. (CU; NI) (Maes; Carvalho, 1989; Hernandez; Henry, 2010), Amaranthus sp. (BR: MG) (Ferreira et al., 2001), Amaranthus spinosus L. (BR: MG; NI) (Maes; Carvalho, 1989; Ferreira et al., 2001); Apiaceae: Daucus carota L. (BR: MG) (Alayo, 1974; Ferreira et al., 2001), Eryngium agavifolium Griseb. (BR: RS) (CoeIho, 2008); Associação entre Poaceae: Brachiaria humidicola (Rendle) Schweick, Brachciaria brizantha (A. Rich.) Stapf e Brachiaria ruziziensis R.Germ. \& C.M.Evrard (BR: ES) (Ruiz, 2014); Asteraceae: Bidens pilosa L. (BR: MG; NI) (Maes; Carvalho, 1989; Ferreira et al., 2001), Dahlia sp. (BR: MG) (Ferreira et al., 2001), Helianthus annuus L. (NI) (Maes; Carvalho, 1989), Helianthus sp. (BR: MG) (Ferreira et al., 2001), Parthenium hysterophorus L. (CU; NI) (Maes; Carvalho, 1989; Wheeler, 2001; Hernandez; Henry, 2010), Parthenium sp. (BR: $M G)$ (Ferreira et al., 2001); Cleomaceae: Cleome sp. (BR: MG; NI) (Maes; CarvaIho, 1989; Ferreira et al., 2001); Convolvulaceae: Ipomoea sp. (CU) (Hernandez; Henry, 2010); Fabaceae: Phaseolus sp. (CU) (Hernandez; Henry, 2010), Phaseolus vulgaris L. (CU) (Alayo, 1974); Malvaceae: Gossypium hirsutum L. (CU) (Alayo, 1974), Gossypium sp. (BR: MG; NI) (Maes; Carvalho, 1989; Ferreira et al., 2001); Rubiaceae: Borreria sp. (BR: MG) (Ferreira; Rossi, 1979), Spermaco- ce verticillata L. (AR; PY) (Logarzo et al., 2005); Solanaceae: Nicotiana tabacum L. (BR: MG) (Ferreira et al., 2001), Solanum lycopersicum L. (CU; NI) (Maes; CarvaIho, 1989; Hernandez; Henry, 2010).

Proba vittiscutis (Stal, 1860): Apiaceae: Coriandrum sativum L. (BR: MG) (UFVB); Asteraceae: Baccharis dracunculifolia DC. (AR; PY) (Logarzo et al., 2005), Baccharis punctulata DC. (AR; PY) (Logarzo et al., 2005), Chromolaena odorata (L.) R. King \& H. Rob. (AR; PY) (Logarzo et al., 2005), Clibadium erosum (Sw.) DC. (NI) (Maes; Carvalho, 1989), Parthenium hysterophorus L. (AR; PY) (Wheeler, 2001; Logarzo et al., 2005)"title" : "Biology of the Plant Bugs (Hemiptera: Miridae, Vernonanthura puberula (Less.) H. Rob. (BR) (UFVB); Fabaceae: Phaseolus vuglaris L. (BR: MG) (Ferreira et al., 2001); Malvaceae: Gossypium herbaceum L. (BR: MG) (Ferreira; Rossi, 1979), Gossypium hirsutum L. (Wheeler, 2001).

Taedia distantina Carvalho, 1954: Euphorbiaceae: Manihot esculenta Crantz (BR: MG) (UFVB).

Taedia guttulosa (Reuter, 1907): Amaranthaceae: Gomphrena haenkeana C. Mart. (AR; PY) (Logarzo et al., 2005).

Taedia nobilitata (Stal, 1860): Solanaceae: Nicotiana tabacum L. (BR: MG) (Ferreira; Rossi, 1979).

Taedia signata Carvalho e Gomes, 1971: Asteraceae: Mikania cordifolia (L. Fil.) (BR: MG) (Ferreira; Rossi, 1979); Lamiaceae: Condea undulata (Schrank) 
Harley \& J.F.B. Pastore (BR: MG) (Ferreira; Rossi, 1979).

Taedia similares Carvalho et Gomes 1971: Araceae (BR: MG) (UFVB).

Taedia stigmosa (Berg, 1878): Araceae (BR: MG) (Ferreira; Rossi, 1979); Asteraceae: Baccharis dracunculifolia (AR; PY) (Logarzo et al., 2005); Malvaceae: Gossypium hirsutum (BR: MG, SP) (UFVB; Silva et al., 1968; Ferreira et al., 2001; Silvie; Thomazoni, 2007); Plantaginaceae: Stemodia verticillata (AR; PY) (Logarzo et al., 2005); Poaceae: Brachiaria ruziziensis (BR: MT) (UFVB); Associação entre Fabaceae: Cajanus Cajan e Poaceae: Eleusine coracana (BR) (UFVB).

Taylorilygus apicalis (Fieber, 1861): Amaranthaceae: Amaranthus sp. (NI) (Maes; Carvalho, 1989), Beta vulgaris L. (BR: MG) (Ferreira et al., 2001), Chenopodium album L. (BR: RS) (Coelho, 2008), Chenopodium sp. (NI) (Maes; Carvalho, 1989), Gomphrena perennis L. (BR: RS) (Coelho, 2008); Apiaceae: Daucus carota L. (BR: MG) (Ferreira et al., 2001), Visnaga daucoides Gaertn. (AR; PY) (Logarzo et al., 2005); Associação entre Poaceae: Brachiaria brizantha (A. Rich) Stapf e Panicum maximum cv. Mombaça Jacq. (BR: ES) (Ruiz, 2014); Asteraceae: Ambrosia sp. (NI) (Maes; Carvalho, 1989), Ambrosia tenuifolia Spreng. (AR; PY) (Logarzo et al., 2005), Anthemis sp. (NI) (Maes; Carvalho, 1989), Aster sp. (NI) (Maes; Carvalho, 1989), Austroeupatorium inulifolium (Kunth) R. King \& H. Rob. (BR:
RS) (Coelho, 2008), Baccharis coridifolia DC. (Logarzo et al., 2005), Baccharis dracunculifolia DC. (AR; PY) (Logarzo et al., 2005), Baccharis punctulata DC. (AR; PY; BR: RS) (Logarzo et al., 2005, CoeIho, 2008), Baccharis salicifolia subsp. salicifolia (Ruiz \& Pav.) Pers. (AR; PY) (Logarzo et al., 2005), Baccharis sp. (NI) (Maes; Carvalho, 1989), Badilloa steetzii (B.L. Rob.) R. King \& H. Rob. (BR: RS) (Coelho, 2008), Barrosoa candolleana (Hook. \& Arn.) R. King \& H. Rob. (AR; PY) (Logarzo et al., 2005), Bidens sp. (NI) (Maes; Carvalho, 1989), Calendula officinalis L. (BR: MG) (UFVB), Chromolaena christieana (Baker) R. King \& H. Rob. (BR: RS) (Coelho, 2008), Chromolaena laevigata (Lam.) R. King \& H. Rob. (BR: RS) (Coelho, 2008), Conyza sp. (AR; PY; NI) (Maes; Carvalho, 1989; Logarzo et al., 2005), Conyza spp. (AR; PY) (Logarzo et al., 2005), Coreopsis sp. (NI) (Maes; Carvalho, 1989), Dracopis sp. (NI) (Maes; Carvalho, 1989), Eclipta sp. (NI) (Maes; Carvalho, 1989), Erigeron sp. (NI) (Maes; Carvalho, 1989), Eupatorium sp. (NI) (Maes; Carvalho, 1989), Flaveria bidentis (L.) Kuntze (BR: RS) (Coelho, 2008), Galinsoga parviflora Cav. (BR: RS) (Coelho, 2008), Gamochaeta pensylvanica (Willd.) Cabrera (AR; PY) (Logarzo et al., 2005), Hapopappus sp. (NI) (Maes; Carvalho, 1989), Helenium sp. (NI) (Maes; Carvalho, 1989), Helianthus annuus L. (NI) (Maes; Carvalho, 1989), Iva sp. (NI) (Maes; Carvalho, 1989), Matricaria sp. (NI) (Maes; Carvalho, 1989), Mikania cordifolia (L. Fil.) (BR: RS) (CoeIho, 2008), Mikania sp. (NI) (Maes; Car- 
valho, 1989), Parthenium hysterophorus L. (CU) (Hernandez; Henry, 2010), Parthenium sp. (NI) (Maes; Carvalho, 1989), Pluchea sp. (NI) (Maes; Carvalho, 1989), Pterocaulon alopecuroides (Lam.) DC. (BR: RS) (Coelho, 2008), Pyrrhopappus carolinianus (Walt.) DC. (BR) (UFVB), Pyrrhopappus sp. (BR) (UFVB), Senecio sp. (NI) (Maes; Carvalho, 1989), Senecio viravira G. Hieronymus (BR: RS) (CoeIho, 2008), Solidago chilensis Meyen (NI; PY; AR) (Maes; Carvalho, 1989; Logarzo et al., 2005), Solidago sp. (NI) (Maes; Carvalho, 1989), Sonchus oleraceus L. (BR: RS) (Coelho, 2008), Sonchus sp. (NI) (Maes; Carvalho, 1989), Spilanthes sp. (NI) (Maes; Carvalho, 1989), Stevia multiaristata (BR: RS) (Coelho, 2008), Stomatanthes oblongifolius (Spreng.) $\mathrm{H}$. Rob. (BR: RS) (Coelho, 2008), Urolepis hecatantha (DC.) (BR: RS) (Coelho, 2008), Wedelia sp. (BR: RS) (Coelho, 2008), Xanthium sp. (NI) (Maes; CarvaIho, 1989); Brassicaceae: Lepidium sp. (BR) (UFVB), Lepidium virginicum L. (BR: RS) (UFVB); Calyraceae: Acicarpha tribuloides Juss. (AR; PY) (Logarzo et al., 2005); Convolvulaceae: Ipomoea batatas (L.) Lam. (NI) (Maes; Carvalho, 1989); Euphorbiaceae: Croton sp. (NI) (Maes; Carvalho, 1989), Euphorbia sp. (NI) (Maes; Carvalho, 1989); Fabaceae: Arachis pintoi (BR: RS, MT) (UFVB), Cassia sp. (NI) (Maes; Carvalho, 1989), Medicago sp. (NI) (Maes; Carvalho, 1989), Vicia sp. (NI) (Maes; Carvalho, 1989); Geraniaceae: Geranium sp. (NI) (Maes; Carvalho, 1989); Lamiaceae: Condea undulata (Schrank) Harley \& J.F.B.Pasto- re (BR) (Coelho, 2008), Hyptis lappacea Benth. (BR: RS) (Coelho, 2008), Lamium sp. (NI) (Maes; Carvalho, 1989); Lythraceae: Lythrum sp. (NI) (Maes; Carvalho, 1989); Onagraceae: Ludwigia sp. (NI) (Maes; Carvalho, 1989), Oenothera sp. (NI) (Maes; Carvalho, 1989); Polygonaceae: Polygonum sp. (NI) (Maes; CarvaIho, 1989), Portulaca sp. (NI) (Maes; Carvalho, 1989), Rumex sp. (NI) (Maes; Carvalho, 1989); Ranunculaceae: Ranunculus sp. (NI) (Maes; Carvalho, 1989); Salicaceae: Salix sp. (NI) (Maes; Carvalho, 1989); Solanaceae: Solanum sp. (NI) (Maes; Carvalho, 1989); Verbenaceae: Glandularia sp. (BR: RS) (Coelho, 2008), Verbena sp. (NI) (Maes; Carvalho, 1989).

Trigonotylus ruficornis (Geoffroy, 1785): Poaceae (BR: SP) (Silva et al., 1968).

\section{Resthenini}

Platytylus bicolor (Le Peletier e Serville, 1825): Rutaceae: Citrus aurantium L. (BR) (UFVB), Citrus sp. (BR: SP) (Silva et al., 1968).

Prepops atroluteus (Walker, 1873): Melastomataceae: Pleroma trichopodum DC. (BR: PR) (Coelho, 2012).

Prepops circummaculatus (Stal, 1854): Onagraceae: Ludwigia octovalvis (Jacq.) Raven (BR: PR) (Capriles, 1969).

Prepops cruciferus (Berg, 1878): Aquifoliaceae: Ilex cornuta Lindl. \& Paxton (CU) (Hernandez; Henry, 2010); Onagraceae: Ludwigia octovalvis (Jacq.) Raven (CU; US) (Henry, 1990; Hernandez; Henry, 2010), Ludwigia peruviana (L.) 
Hara (CU; US) (Henry, 1990; Hernandez; Henry, 2010).

Prepops flavicostus (Berg, 1884): Fabaceae: Phaseolus vulgaris L. (BR) (UFVB).

Prepops flavoniger (Stal, 1860): Fabaceae: Phaseolus vulgaris L. (BR: MG) (UFVB).

Prepops zetterstedti (Stal, 1860): Asteraceae: Sonchus sp. (BR: MG) (Ferreira; Rossi, 1979), Brassicaceae: Raphanus raphanistrum L. (BR: MG) (Ferreira et al., 2001); Convolvulaceae: Ipomoea batatas (L.) Lam. (BR: MG) (Ferreira et al., 2001); Fabaceae: Phaseolus vulgaris $\mathrm{L}$. (BR: MG) (Ferreira et al., 2001).

\section{Stenodemini}

Collaria capixaba Carvalho e Fontes, 1981: Poaceae (BR: ES) (Carvalho; Fontes, 1981).

Collaria guaraniana Carvalho e Fontes, 1981: Poaceae (BR: PR) (Carvalho; Fontes, 1981).

Collaria husseyi Carvalho, 1955: Poaceae (BR: MG) (Ferreira et al., 2001).

Collaria oleosa (Distant, 1883): Apiaceae: Coriandrum sativum L. (BR: MG) (UFVB); Brassicaceae: Raphanus raphanistrum L. (BR: MG) (Ferreira; Rossi, 1979); Cucurbitaceae: Cucumis sp. (BR) (UFVB); Dioscoreaceae: Dioscorea trifida L.f. (Wheeler, 2001); Fabaceae: Phaseolus vulgaris $\mathrm{L}$. (BR: MG) (Ferreira et al., 2001), Vigna unguiculata (L.) Walp. (BR) (UFVB); Poaceae: Andropogon gayanus
Kunth (BR: MG) (Ferreira; Rossi, 1979), Andropogon spp. (CU) (Hernandez; Henry, 2010), Avena sativa L. (BR: MG) (Ferreira; Rossi, 1979), Brachiaria arrecta (Hack.) Stent (BR: BA) (Menezes, 1986), Brachiaria brizantha (A. Rich.) (Ruiz, 2014) (BR: ES), Brachiaria decumbens Stapf (BR: ES) (Ruiz, 2014), Brachiaria mutica (Forssk.) Stapf (BR: ES, MG) (Ferreira; Rossi, 1979; Ruiz, 2014), Brachiaria ruziziensis R.Germ. \& C.M.Evrard (BR: ES) (Ruiz, 2014), Cynodon spp. (BR: ES) (Ruiz, 2014), Digitaria abyssinica (Hochst. ex A. Rich.) Stapf (BR) (UFVB), Digitaria eriantha Steud. (BR: MG; CU) (Ferreira; Rossi, 1979; Hernandez; Henry, 2010), Digitaria sanguinalis (L.) Scop. (Hernandez; Henry, 2010), Digitaria sp. (BR: MG) (Ferreira et al., 2001), Echinochloa colona (L.) Link (BR: BA) (Menezes, 1986), Eriochloa polystachya Kunth (CU) (Hernandez; Henry, 2010), Hordeum vulgare L. (BR: MG) (Ferreira; Rossi, 1979), Oryza sativa L. (BR: MG; CU) (Ferreira; Rossi, 1979; Hernandez; Henry, 2010; Wheeler, 2001), Panicum maximum cv. Mombaça Jacq. (BR: ES) (Ruiz, 2014), Panicum maximum cv. Tanzania Jacq. (BR: ES) (Ruiz, 2014), Panicum sp. (BR: MG; CU) (Ferreira et al., 2001; Hernandez; Henry, 2010), Paspalum conjugatum P. Beauv. (BR: BA) (Menezes, 1986), Paspalum notatum Fluggé (BR: BA) (Menezes, 1986), Poaceae (BR: MG) (Silva et al., 1968; Ferreira et al., 2001), Setaria setosa (Sw.) P. Beauv. (BR: MG) (Ferreira; Rossi, 1979), Setaria sp. (BR: MG) (Fer- 
reira et al., 2001), Sorghum bicolor (L.) Moench (BR: MG) (Ferreira et al., 2001), Sorghum sp. (Wheeler, 2001), Stenotaphrum dimidiatum (L.) Brongin (BR: MG) (Ferreira; Rossi, 1979), Triticum aestivum L. (BR: MG) (Ferreira; Rossi, 1979), Triticum sp. (BR: MG) (Ferreira et al., 2001), Zea mays L. (BR: MG) (Ferreira; Rossi, 1979; Ferreira et al., 2001).

Collaria scenica (Stal, 1859): Poaceae: Avena sativa L. (BR: MG) (UFVB; Ferreira et al., 2001), Avena strigose Schreb. (BR: MG, PR) (UFVB), Brachiaria mutica (Forssk.) Stapf (BR) (Silva et al., 1968), Cynodon dactylon (L.) Pers. (BR: PR) (UFVB), Echinochloa colona (L.) Link (BR: MG) (UFVB; Ferreira et al., 2001), Festuca arundinacea Schreb (BR: PR) (UFVB; Carlessi et al., 1999), Oryza sativa L. (BR: MG) (Ferreira et al., 2001), Cenchrus clandestinus (Hochst. Ex Chiov.) Morrone (BR: MG) (Ferreira et al., 2001), Phalaris aquatic L. (BR: PR) (UFVB), Stenotaphrum secundatum (Walter) Kuntze (BR: MG) (Ferreira et al., 2001), Triticum aestivum L. (BR) (Silva et al., 1968), Triticum sp. (BR: MG) (Ferreira et al., 2001), Zea mays L. (BR: MG) (Silva et al., 1968; Ferreira et al., 2001).

Cynodonmiris costicollis (Berg, 1878): Poaceae: Cynodon spp. (BR) (UFVB).

Dolichomiris linearis Reuter, 1882: Poaceae: Aristida sp. (BR: MG, BA, MT, SC; NI; PY) (Carvalho; Hussey, 1954; Carvalho, 1975b; Maes; Carvalho, 1989; Ferreira et al., 2001), Brachiaria brizantha (A.Rich.) Stapf (Ruiz, 2014) (BR:
ES), Brachiaria decumbens Stapf (BR: ES) (Ruiz, 2014), Chloris sp. (BR: MG) (Ferreira et al., 2001), Cynodon spp. (BR: ES) (Ruiz, 2014), Chloris inflata Link (NI; BR: BA, MT, SC) (Carvalho, 1975a, b; Maes; Carvalho, 1989), Melinis repens (Willd.) Zizka (CU) (Alayo, 1974; Hernandez; Henry, 2010).

Trigonotylus tenuis Reuter, 1893: Cyperaceae: Cyperus sp. (BR) (UFVB); Fabaceae: Phaseolus vulgaris L. (NI) (Maes; Carvalho, 1989); Juglandaceae: Juglans sp. (NI) (Maes; Carvalho, 1989); Pedaliaceae: Sesamum indicum L. (NI) (Maes; Carvalho, 1989); Poaceae: Bromus sp. (NI) (Maes; Carvalho, 1989), Chloris inflate Link (BR: BA, MT, SC; NI) (Carvalho, 1975a, b), Cynodon dactylon (L.) Pers (CU; NI) (Alayo, 1974; Maes; Carvalho, 1989; Hernandez; Henry, 2010), Cynodon spp. (Ruiz, 2014), Digitaria sp. (Maes; Carvalho, 1989), Eleusine coracana (L.) Gaertn (BR: MT) (UFVB), Eleusine indica (L.) Gaertn. (CU; $\mathrm{NI}$; PR) (Capriles, 1969; Maes; Carvalho, 1989; Hernandez; Henry, 2010), Eragrostis spp.(BR: MG) (UFVB), Hordeum sp. (Maes; Carvalho, 1989), Leptochloa sp. (NI) (Maes; Carvalho, 1989), Medicago sativa L. (NI) (Maes; Carvalho, 1989), Oryza sativa L. (BR) (Vivas et al., 2005), Setaria sp. (NI) (Maes; Carvalho, 1989), Sorghum bicolor (L.) Moench (NI) (Maes; Carvalho, 1989), Zea mays L. (NI) (Maes; Carvalho, 1989). 


\section{Phylinae}

Phylini

Alvarengamiris alvarengai Carvalho, 1991: Arecaceae: Mauritia flexuosa L.f. (BR: RO, PR) (Costa; Couturier, 2002).

Anomalocornis rondonienses CarvaIho, 1984: Arecaceae: Arecaceae (BR: AM) (Costa; Couturier, 2012), Astrocaryum urostachys Burret (BR: RO, PR) (Costa; Couturier, 2012), Oenocarpus bataua Mart (BR: RO, PR) (Costa; Couturier, 2012).

Anomalocornis tucuruiensis CarvaIho, 1984: Arecaceae: Astrocaryum javarense (Trail) Drude (Costa; Couturier, 2012), Astrocaryum urostachys Burret (BR) (Costa; Couturier, 2012).

Moissonia cuneata (Stal, 1860): Arecaceae; Convolvulaceae: Ipomoea batatas (L.) Lam. (BR: MG) (Ferreira; Rossi, 1979; Ferreira et al., 2001); Fabaceae: Crotalaria sp. (BR: MG) (Ferreira et al., 2001); Poaceae: Eleusine sp. (BR: MG) (Ferreira et al., 2001), Setaria sp. (BR: MG) (Ferreira et al., 2001), Sorghum bicolor (L.) Moench (BR: MG) (Ferreira; Rossi, 1979; Ferreira et al., 2001); Thymelaeaceae: Daphne sp. (BR: MG) (Ferreira et al., 2001).

Platyscytisca bergmannae Costa e Henry, 1999: Moraceae: Ficus sp. (BR: SP) (Henry; Costa, 2003).

Platycytus decempunctatus (CarvaIho, 1945): Solanaceae: Solanum cernuum Vell. (BR: MG) (Carvalho, 1945; Ferreira; Rossi, 1979; Pires et al., 2008).
Platycytus montei (Carvalho, 1945): Euphorbiaceae: Croton floribundus Spreng (BR: MG) (Ferreira et al., 2001), Croton sp. (BR: MG) (Ferreira; Rossi, 1979; Ferreira et al., 2001); Solanaceae: Solanum bullatum Vell. (BR: MG) (Ferreira et al., 2001), Solanum donianum Walp. (BR: MG) (Ferreira et al., 2001), Solanum sp. (BR: MG) (Ferreira et al., 2001).

Platycytus rufoscutellatus (Carvalho, 1945): Euphorbiaceae: Croton floribundus Spreng. (BR: MG) (Ferreira et al., 2001), Croton sp. (BR: MG) (Ferreira; Rossi, 1979), Tragia sp. (BR: MG) (Silva et al., 1968; Ferreira; Rossi, 1979; Ferreira et al., 2001).

Rhinacloa basalis (Reuter, 1907): Amaranthaceae: Amaranthus dubius Mart. (Schuh; Schwartz, 1985); Asteraceae: Bidens pilosa L. (BR) (UFVB); Fabaceae: Cajanus cajan (L.) Millsp. (NI) (Maes; Carvalho, 1989), Enterolobium cyclocarpum (Jacq.) Griseb. (CU) (Hernandez; Henry, 2010).

Rhinacloa clavicornis (Knight, 1927): Anacardiaceae: Schinus terebinthifolius Raddi (CU) (Hernandez; Henry, 2010); Associação entre Poaceae: Sorghum bicolor (L.) Moench e Brachiaria ruziziensis R.Germ \& C.M.Evrard (BR: MT) (UFVB); Asteraceae (NI) (Maes; Carvalho, 1989); Fabaceae: Crotalaria juncea L. (AU) (Donnelly, 2000), Phaseolus lunatus L. (BR) (UFVB), Phaseolus vulgaris L. (NI) (Maes; Carvalho, 1989); Lauraceae: Persea Americana Mill. (Wheeler, 2001); Malvaceae: Gossypium herbaceum L. (BR: MG) (Ferreira; Rossi, 1979), Gos- 
sypium hirsutum L. (BR: MG) (Ferreira et al., 2001); Scrophulariaceae: Budleja sp. (AU) (Donnelly, 2000), Budleja sessiliflora Kunth (BR) (UFVB); Solanaceae: Solanum melongena L. (BR) (Silva et al., 1968).

Rhinacloa forticornis Reuter, 1876: Asteraceae: Ambrosia artemisioides Meyen \& Walp. (AU) (Donnelly, 2000), Franseria sp. (NI) (Maes; Carvalho, 1989), Malacothris sp. (NI) (Maes; CarvaIho, 1989); Fabaceae: Acacia constricta Benth. (BR) (UFVB), Cajanus cajan (L.) Millsp. (NI; BR: MG) (Maes; Carvalho, 1989; Ferreira et al., 2001), Crotalaria juncea L. (NI; BR: MG) (Maes; Carvalho, 1989; Ferreira et al., 2001), Medicago sativa L. (NI; BR: MG) (Maes; CarvaIho, 1989; Ferreira et al., 2001), Mimosa sp. (BR: MG) (Ferreira et al., 2001), Mimosa tenuiflora (Willd.) Poir. (NI; BR: MG) (Carvalho, 1975a; Maes; Carvalho, 1989), Phaseolus vulgaris L. (NI; BR: MG) (Maes; Carvalho, 1989; Ferreira et al., 2001); Malvaceae: Gossypium barbadense L. (AU) (Donnelly, 2000), Gossypium hirsutum L. (NI) (Butler, 1965; Maes; Carvalho, 1989), Gossypium sp. (BR: MG) (Ferreira et al., 2001), Sphaeralcea sp. (NI) (Maes; Carvalho, 1989); Polygonaceae: Eriogonum sp. (NI) (Maes; Carvalho, 1989); Rosaceae: Purshia mexicana (D. Don) S.L. Welsh (BR) (UFVB); Solanaceae: Solanum tuberosum L. (NI) (Maes; Carvalho, 1989); Ulmaceae: Ulmus sp. (NI) (Maes; Carvalho, 1989).

Rhinacloa pallidipes Maldonado, 1969: Anacardiaceae: Schinus sp. (NI)
(Maes; Carvalho, 1989), Schinus terebinthifolius Raddi (US) (Henry, 1984); Fabaceae: Cajanus cajan (L.) MIllsp. (BR: MG) (Ferreira et al., 2001).

Spanagonicus argentinus (Berg, 1883): Asteraceae: Ambrosia acanthicarpa Hook. (BR) (UFVB), Artemisia sp. (BR: MG) (Ferreira et al., 2001); Fabaceae: Phaseolus vulgaris L. (BR: MG) (Ferreira et al., 2001); Malvaceae: Gossypium sp. (BR: MG) (Ferreira et al., 2001); Poaceae: Oryza sp. (BR: MG) (Ferreira et al., 2001).

Tytthus femoralis Henry, 2012: Solanaceae: Solanum tuberosum L. (BR: MG) (UFVB).

Tytthus neotropicalis (Carvalho, 1954): Poaceae: Cynodon spp. (BR: ES) (Ruiz, 2014).

Tytthus parviceps (Reuter, 1890): Cyperaceae: Cyperus sp. (EG; NI) (Linnavuori, 1964; Maes; Carvalho, 1989); Fabaceae: Medicago sativa L. (US) (Henry, 2012), Phaseolus sp. (CU) (Hernandez; Henry, 2010), Phaseolus vulgaris L. (NI) (Maes; Carvalho, 1989); Poaceae: Oryza sativa L. (CU; NI) (Alayo, 1974; Maes; Carvalho, 1989; Hernandez; Henry, 2010), Oryza sp. (Henry, 2012), Saccharum officinarum L. (US) (Henry, 2012), Sporobolus alterniflorus (Loisel.) P.M. Peterson \& Saarela (US) (Henry, 2012), Sporobolus virginicus (L.) Kunth (US) (Henry, 2012), Zea mays L. (NI) (Maes; Carvalho, 1989). 


\section{Pilophorini}

Sthenaridea carmelitana Carvalho, 1945: Associação entre Poaceae Panicum maximum Jacq. e Eleusine coracana (L.) Gaertn (BR: MT) (UFVB); Associação entre Poaceae: Brachiaria brizantha (A. Rich) Stapf, Brachiaria decumbens Stapf, Brachiaria ruziziensis R.Germ. \& C.M.Evrard, Cynodon spp. e Panicum maximum cv. Mombaça Jacq. (BR: ES) (Ruiz, 2014); Associação entre Poaceae: Brachiaria ruziziensis R.Germ. \& C.M.Evrard e Panicum maximum Jacq. (BR: MT) (UFVB); Cyperaceae: Cyperus rotundus L. (BR: MG, MT, RJ, RS, SC) (Ferreira et al., 2001; Coelho, 2008), Cyperus luzulae (L.) Retz. (Schuh; Schwartz, 1985); Fabaceae: Cajanus cajan (L.) Millsp. (PR) (Capriles, 1969); Poaceae: Echinochloa colona (L.) Link (BR: MG, RS) (Ferreira et al., 2001; Coelho, 2008), Eleusine coracana (L.) Gaertn. (BR: RS) (UFVB; Coelho, 2008), Oryza sativa (BR) (UFVB), Panicum maximum Jacq. (BR: MG) (Ferreira et al., 2001; Coelho, 2008), Sorghum bicolor (L.) Moench (BR: MG, RS) (Ferreira et al., 2001; Coelho, 2008), Zea mays L. (BR: MG, RS) (Ferreira et al., 2001; CoeIho, 2008).

Sthenaridea carvalhoi Schuh e Schwartz, 1988: Poaceae: Oryza sativa L. (BR) (UFVB).
Sthenaridea vulgaris (Distant, 1893): Cyperaceae: Cyperus luzulae (L.) Retz. (BR) (UFVB); Pinaceae: Pinus sp. (BR) (UFVB); Poaceae: Sorghum sp. (CU) (Hernandez; Henry, 2010), Zea mays L. (BR) (UFVB).

*Abreviações dos Países: Argentina (AR); Australia (AU); Brazil (BR); Canada (CA); Chile (CL); Colombia (CO); Costa Rica (CR); Cuba (CU); Ecuador (EC); Egypt (EG); Estados Unidos (US); France (FR); Georgia (GE); Guatemala (GT); Guyana (GY); Mexico (MX); Nicaragua (NI); Panama (PA); Paraguay (PY); Peru (PE); Puerto Rico (PR); United Kingdom (UK); Uruguay (UY); Venezuela (VE).

*Abreviações dos estados brasileiros: Acre (AC); AL (Alagoas); AP (Amapá); AM (Amazonas); Bahia (BA); Ceará (CE); Distrito Federal (DF); Espírito Santo (ES); Goiás (GO); Maranhão (MA); Mato Grosso (MT); Mato Grosso do Sul (MS); Minas Gerais (MG); Pará (PA); Paraíba (PB); Paraná (PR); Pernambuco (PE); Piauí (PI); Rio de Janeiro (RJ); Rio Grande do Norte (RN); Rio Grande do Sul (RS); Rondônia (RO); Roraima (RR); Santa Catarina (SC); São Paulo (SP); Sergipe (SE); Tocatins (TO). 


\section{Referências}

ABREU, J. M. Mirídeos neotropicais associados ao cacaueiro. In: LAVABRE E. M. (Org.) Les mirides du cacaoyer. Paris: Institut Français du Cafe et du Cacao, 1977. p. 85-106.

ALAYO, D. P. Los Hemipteros de Cuba. Parte XIII. Familia Miridae. Torreia, v. 32, p. 1-41, 1974.

BAUTISTA, L.; CARDONA, J.; SOTO, A. Distribución espacial de Collaria scenica (Hemiptera: Miridae) y Hortensia similis (Hemiptera: Cicadellida) en Valles Andinos.- Boletín Científico Museo de Historia Natural, v. 17, p. 75-84, 1974.

BOTI, J. B.; MADALON, F. Z.; OLIVEIRA, B. R.; HADDADE, I. R. Insetos provocadores de danos em folhas, flores e frutos da goiabeira (Psidium guajava L., Myrtaceae) nos pomares conduzidos em sistema de cultivo convencional e orgânico, no município de Santa Teresa- ES. Natureza online, v. 14, p. 40-44, 2016.

CAPRILES, J. M. The Miridae of Puerto Rico (Insecta, Hemiptera). Agricultural Experiment Station Technical Paper, University of Puerto Rico, Río Piedras, PR, 1969.

CARLESSI, L. R. G.; CORSEUIL, E.; SALVADORI, J. R. Aspectos biológicos e morfométricos de Collaria scenica (Stal) (Hemiptera: Miridae) em trigo. Anais da Sociedade Entomológica do Brasil, v. 28, p. 65-73, 1999.

CARTER, W. Insects in relation to plant disease. 2nd ed.Wiley, New York, USA, 1973.

CARVALHO, J. C. M. Mirideos neotropicais: sôbre o gênero Sericophanes Reuter, com descrição de uma nova espécie (Hemiptera). Revista Brasileira de Biologia, v. 4, p. 517-530, 1944a.

CARVALHO, J. C. M. Mirideos neotropicais: sobre os gêneros Jobertus Distant, Parachius Distant e Bergroth (Hemiptera). Revista de Entomologia, v. 15, p. 162-171, 1944b.

CARVALHO, J. C. M. Mirideos neotropicais: Gêneros Diaphnidia Uhler, Hyaliodes Reuter, Hyaliodocoris Knight, Sinervius Stål e Spartacus Distant, com descrições de espécies novas. Boletim do Museu Nacional do Rio de Janeiro, v. 36, p. 1-7, 1945.

CARVALHO, J. C. M. Mirideos neotropicais, XXIII: Um gênero e três espécies novas colecionadas em Araceae e Bromeliacea (Hemiptera). Boletim do Museu Nacional do Rio de Janeiro, v. 61, p. 1-6, 1946.

CARVALHO, J. C. M. Mirideos neotropicais, XXXV: Gêneros Corcovadocola n. g., Guanabarea n. g. e Caulotops Bergroth (Hemiptera). Revista Brasileira de Biologia, p. 522-533, 1948.

CARVALHO, J. C. M. Mirideos neotropicais, XXXVIII: Descrições de três espécies novas (Hemiptera). Anais da Academia Brasileira de Ciências, v. 22, p. 19-24, 1950.

CARVALHO, J. C. M. Neotropical Miridae, 68: Genus Eurychilella Reuter wtih description of four new species (Hemiptera). Revista Brasileira de Biologia, v. 13, p. 347-353, 1953.

CARVALHO, J. C. M. Neotropical Miridae, LXIV: New bugs of the subfamily Cylapinae (Hemiptera). Proceedings of the United States National Museum, v. 103, p. 621-632, 1955.

CARVALHO, J. C. M. Mirideos neotropicais, CXLVI: Gênero Monalonion H.-S., 1853 (Hemiptera). Anais da Academia Brasileira de Ciências, v. 44, p. 119-143, 1972.

CARVALHO, J. C. M. Neotropical Miridae, CLXXXVIII: On the genera Dolichomiris Reuter, Megaloceroea Fieber, Stenodema Laporte, Trigonotyliscus n. gen. and Trigonotylus Fieber (Hemiptera). Revista Brasileira de Biologia, v. 35, p. 1-121, 1975a.

CARVALHO, J. C. M. Mirideos neotropicais, CXCIII: Sôbre algumas espécies que ocorrem nas caatingas brasileiras (Hemiptera). Revista Brasileira de Biologia, v. 35, p. 451-459, 1975b. 
CARVALHO J.C.M. Mirideos neotropicais, CCCIV: Novos gêneros e espécies do Brasil (Hemiptera). Revista Brasileira de Biologia, v. 49, p. 443-460, 1989.

CARVALHO, J. C. M.; COSTA, L. A. A. The genus Fulvius from the Americas (Hemiptera: Miridae). Anales del Instituto de Biologia de la Universidad Nacional Autonoma del Mexico, v. 65, p. 63-135, 1994.

CARVALHO, J. C. M.; DRAKE, C. J. A new genus and two new species of Neotropical Dicyphinae (Hemiptera). Revista Brasileira de Biologia, v. 3, p. 87-89, 1943.

CARVALHO, J. C. M.; FONTES, A. V. Mirídeos neotropicais CCXXV: Revisão do gênero Collaria Provancher no continente Americano (Hemiptera). Experientiae, p. 27: 11-46, 1981.

CARVALHO, J. C. M.; HUSSEY, R. F. Neotropical Miridae, LVII: On a collection from Paraguay, with descriptions of three new species. Occasional Papers of the Museum of Zoology University of Michigan, v. 552, p. 1-11, 1954.

CARVALHO, J. C. M.; ROSAS, A. F. Mirídeos neotropicais, XCl: Uma tribo e dois gêneros novos (Hemiptera). Revista Brasileira de Biologia, p. 427-432, 1962

COELHO, L. A. Miridofauna (Hemiptera: Heteroptera: Miridae) do Rio Grande do Sul, Brasil. Viçosa: UFV, 2008. 175p.

COELHO, L. A. Contribuição à taxonomia e biogeografia do gênero Prepops Reuter, 1905 (Hemiptera: Miridae). Viçosa: UFV, 2012. 142p.

COSTA, R. G. Alguns insetos e outros pequenos animais que danificam plantas no Rio Grande do Sul. Porto Alegre, Secção de Informações e Propangas Agrícolas 296, 1958.

COSTA, L. A. A.; COUTURIER, G. Le genre Anomalocornis Carvalho et Wygodzinsky 1945 (Heteroptera: Miridae: Phylinae), révision taxonomique, description d'une espèce nouvelle et affinités avec les palmiers néotropicaux. Annales de la Société Entomologique de France, v. 48, p. 323-341, 2012.

DAY, W. H.; SAUNDERS, L. B. Abundance of the Garden Fleahopper (Hemiptera: Miridae) on Alfalfa and Parasitism by Leiophron uniformis (Gahan) (Hymenoptera: Braconidae). Journal of Economic Entomology, v. 83, n. 1, p. 101-106, 1990.

DONNELLY, G. P. Biology and host specificity of Rhinacloa callicrates Herring (Hemiptera: Miridae) and its introduction and establishment as a biological control agent of Parkinsonia aculeata L. (Caesalpiniaceae) in Australia. Australian Journal of Entomology, v. 39, p. 89-94, 2000.

FERREIRA, P. S. F. Descrição do macho e primeiro registro para o Brasil de Termatophylidea opaca Carvalho (Heteroptera: Miridae: Termatophylini). Anais da Sociedade de Entomologia do Brasil, v. 22, p. 485-490, 1993.

FERREIRA, P. S. F. Miridae. In: BRANDÃO, C. R. F.; CANCELLO, E. M (Org.). Invertebrados terrestres, Biodiversidade do estado de São Paulo. Síntese do conhecimento ao final do século XX. São Paulo: FAPESP, 1999. p. 93-100.

FERREIRA, P. S. F.; SILVA, E. R.; COELHO, L. B. N. Miridae (Heteroptera) fitófagos e predadores de Minas Gerais, Brasil, com ênfase em espécies com potencial econômico. Iheringia, Série Zoológica, v. 91, p. 159-169, 2001.

FERREIRA, P. S. F.; COSTA, L. A. A.; COELHO, L. A. Redescription, new records, and host plant for Sysinas centralis Distant (Hemiptera: Heteroptera: Miridae). Zootaxa, v. 1581, p. 45-52, 2007. 
FERREIRA, P. S. F.; UCELI, L. F.; COELHO, L. A.; PIROVANI, V. D. Description of a new species Perissobasis heroni (Hemiptera: Heteroptera: Deraeocorinae), found on Coffea arabica, and with key to Neotropical species of the genus. Zootaxa, v. 58, p. 50-58, 2009.

FERREIRA, P. S. F.; HENRY, T. J.; COELHO, L. A. Plant bugs (Miridae). In: PANIZZI, A. R.; GRAZIA, J. (Org). True Bugs (Heteroptera) of the Neotropics. Springer Netherlands, 2015. p. 237-286.

FERREIRA, P. S. F.; HENRY, T. J.; COELHO, L. A. Miridae. Catálogo Taxonômico da Fauna do Brasil, 2018. Disponível em: http://fauna.jbrj.gov.br/fauna/faunadobrasil/1567. Acesso em: 17 out. 2017.

FERREIRA, P. S. F.; ROSSI, D. Catálogo das espécies de miridae (Hemiptera) de Viçosa, estado de Minas Gerais, Brasil. Experientiae, v. 25, p. 131-157, 1979.

FERREIRA, P. S. F.; SILVA, E. R.; COELHO, L. B. N. Miridae (Heteroptera) fitófagos e predadores de Minas Gerais, Brasil, com ênfase em espécies com potencial econômico. Iheringia, Série Zoológica, v. 91, p. 159-169, 2001.

FERREIRA, P. S. F.; UCELI, L. F.; COELHO, L. A.; PIROVANI, V. D. Description of a new species Perissobasis heroni (Hemiptera: Heteroptera: Deraeocorinae), found on Coffea arabica, and with key to Neotropical species of the genus. Zootaxa, v. 2127, p. 50-58, 2009.

GASSEN, D. N. Manejo de pragas associadas à cultura do milho. Passo fundo, Aldeia Norte: Embrapa Circular Técnica, 134, 1996.

GRANDI, T. S. M.; TRINDADE, J. A.; PINTO, M. J. F.; FERREIRA, L. L.; CATELLA, A. C. Plantas medicinais de Minas Gerais, Brasil. Acta Botanica Brasilica, v. 2, n. 40, 1989.

HEIDEN, G.; BARBIERI, R. L. Í. A.; REGINA, E.; STUMPF, T. Considerações sobre o uso de plantas ornamentais nativas. Revista brasileira de horticultura ornamental, v. 12, p. 2-7, 2006.

HENRY, T. J. New United States records for two Heteroptera: Pellaea stictica (Pentatomidae) and Rhinacloa pallidipes (Miridae). Proceedings of the Entomological Society of Washington, v. 86, p. 519520,1984

HENRY, T. J. Prepops cruciferus (Heteroptera: Miridae): A Neotropical plant bug newly discovered in the United States: taxonomic notes, host plant, and distribution. Annals of the Entomological Society of America, v. 83, p. 15-17, 1990.

HENRY, T. J. Biodiversity of the Heteroptera. In: FOOTTIT, R. G.; ADLER, P. H. (Org). Insect biodiversity: science and society. Oxford: Wiley-Blackwell Publishing Ltd., 2009. p. 223-263.

HENRY, T. J. Revision of the plant bug genus Tytthus (Hemiptera, Heteroptera, Miridae, phylinae). ZooKeys, v. 220, p. 1-114, 2012.

HENRY, T. J.; COSTA, L. A. A. Review of the Neotropical phyline genus Platycytisca (Hemiptera: Heteroptera: Miridae), with the description of a new species from Bahia, Brazil. Journal of the New York Entomological Society, v. 111, p. 120-125, 2003.

HENRY, T. J.; SMITH, C. L. An annotated list of the Miridae of Georgia (Hemiptera-Heteroptera). Journal of the Georgia Entomological Society, v. 14, p. 212-220, 1979.

HENRY, T. J.; WHEELER, A. G. Family Miridae Hahn, 1833 (= Capsidae Burmeister, 1835). The plant bugs. In: HENRY, T. J.; FROESCHNER, R. C. (Org). Catalog of the Heteroptera, or True Bugs, of Canada and the Continental United States. Leiden, Netherlands: E. J. Brill., 1988. P. 251-507.

HERNANDEZ, L. M.; HENRY, T. J. The plant bugs, or Miridae (Hemiptera: Heteroptera), of Cuba. Pensoft Series Faunistica, 2010. 
HSIAO, T. Y. The genus Eccritotarsus Stål, with descriptions of a new genus and two new species (Hemiptera, Miridae). Proceedings of the Entomological Society of Washington, p. 59-62, 1947.

HSIAO, T. Y.; SAILER, R. I. The orchid bugs of the genus Tenthecoris Scott (Hemiptera: Miridae). Journal of the Washington Academy of Sciences, v. 37, p. 64-72, 1947.

KALVELAGE, H. Collaria scenica (Stal, 1859) (Hemiptera, Miridae): praga de gramíneas forrageiras na região do Planalto Catarinense, Brasil. Anais da Sociedade Entomológica do Brasil, p. 221-222, 1988.

KELTON, L. A. Lectotype designation for Idolocoris agilis, and descriptions of three new species of Dicyphus Fieber from North America (Heteroptera: Miridae). Canadian Entomologist, v. 112, p. 387-392, 1980a.

KELTON, L. A. The insects and arachnids of Canada. Part8. The plant bugs of the prairie provinces of Canada. Heteroptera: Miridae. Ontario: Agriculture Canada Research Branch Publication, Publication 1703 1980b.

LINDBERG, H. Hemiptera Heteroptera from Newfoundland, collected by the swedish-finnish expedition of 1949 and 1951. Acta Zoologica, v. 96, p. 1-25, 1958.

LOGARZO, G. A.; WILLIAMS, L.; CARPINTERO, D. L. Plant Bugs (Heteroptera: Miridae) Associated with roadside habitats in Argentina and Paraguay: Host Plant, Temporal, and Geographic Range Effects. Annals of the Entomological Society of America, v. 98, p. 694-702, 2005.

LÓPEZ, S. N.; ROJAS, F. A.; VELÁSQUEZ, V. V.; CAGNOTTI, C. Biology of Tupiocoris cucurbitaceus (Hemiptera: Miridae), a predator of the greenhouse whitefly Trialeurodes vaporariorum (Hemiptera: Aleyrodidae) in tomato crops in Argentina. Biocontrol Science and Technology, v. 22, p. 1107-1117, 2012.

MAES, J. M.; CARVALHO, J. C. M. Catalogo de los Miridae (Heteroptera) de Nicarágua. Revista Nicaraguense de Entomologia, v. 6, p. 7-36, 1989.

MELO, M. C.; DELLAPÉ, P. M.; CARPINTERO, D. L.; COSCARÓN, M. C. Reduviidae, Miridae y Lygaeoidea (Hemiptera) recolectados en Colonia Carlos Pellegrini (Esteros de Iberá, Corrientes, Argentina). Revista da Sociedad Entomológica Argentina, v. 63, p. 56-67, 2004.

MENEZES, M. Collaria oleosa (Distant, 1883) (Hemiptera: Miridae), nova praga de gramíneas forraeiras no sudeste da Bahia, Brasil. Centro de Pesquisas do Cacau (CEPEC), v. 2, p. 113-118, 1986.

NAMYATOVA, A. A.; CASSIS, G. Systematic revision and phylogeny of the plant bug tribe Monaloniini (Insecta: Heteroptera: Miridae: Bryocorinae) of the world. Zoological Journal of the Linnean Society, v. 176, p. 36-136, 2016.

OLIVEIRA, M. A. S.; FIALHO, J. F.; ICUMA, I. V. Ocorrência do mirídeo Hyaliodes beckeri predando o percevejo da renda Vatiga illudens em mandioca no Distrito Federal. Embrapa Comunicado Técnico 1-2, 2002.

PIRES, E. M.; GUEDES, R. N. C.; SERRÃO, J. E.; FERREIRA, P. S. F. Seasonal and interpopulational morphometry variation of Platyscytus decempunctatus (Carvalho, 1946) (Heteroptera: Miridae). Biota Neotropica, v. 8, p. 21-28, 2008.

ROSKOV Y et al. 2017. Species 2000 \& ITIS Catalogue of Life, 2017 Annual Checklist. Species 2000: Naturalis, Leiden, the Netherlands. ISSN 2405-884X. Disponível em: http://www.catalogueoflife. org/annual-checklist/2017. Acesso em: 17 out. 2017.

RUIZ, M. C. V. Heteroptera fitófagos e predadores em pastagens do estado do Espírito Santo, Brasil. Viçosa: UFV, 2014. 
SCHUH, R. T. Pretarsal structure in the Miridae (Hemiptera) with a cladistic analysis of relationships within the family. American Museum Novitates, v. 36, p. 1-39, 1976.

SCHUH, R. T.; SCHWARTZ, M. D. Revision of the plant bug genus Rhinacloa Reuter with a phylogenetic analysis (Hemiptera: Miridae). Bulletin of the American Museum of Natural History, v. 179, p. 379-470, 1985.

SCHUH, R. T. On-line Systematic Catalog of Plant Bugs (Insecta: Heteroptera: Miridae). Disponível em: http://research.amnh.org/pbi/catalog/. Acesso em: 16 out. 2017.

SILVA, A. G. A.; GONÇALVES, C. R.; GALVÃO, D. M.; GONÇALVES, A. J. L.; GOMES, J.; SILVA, M. N.; SIMONI, L. Quarto catálogo dos insetos que vivem nas plantas do Brasil seus parasitos e predadores. Ministério da Agricultura. Departamento de Defesa e Inspeção Agropecuária, Rio de Janeiro, BR, 1968.

SILVIE, P.; THOMAZONI, D. Manual de identificação das pragas e danos nos sistemas de cultivo do algodão. 38th ed. Cascavel: Boletim Técnico, Coodetec, 2007.

TATARNIC, N. J.; CASSIS, G. The Halticini of the world (Insecta: Heteroptera: Miridae: Orthotylinae): Generic reclassification, phylogeny, and host plant associations. Zoological Journal of the Linnean Society, v. 164, p. 558-658, 2012.

UCELI, L. F.; FERREIRA, P. S. F.; PIROVANI, V. D. Review of Pachymeroceroides genus with the description of a new species (Hemiptera: Heteroptera: Miridae: Bryocorinae). Zootaxa, v. 2174, p. 3744, 2009.

VAN LENTEREN, J. C.; HEMERIK, L.; LINS, J. C.; BUENO, V. H. Functional responses of three neotropical mirid predators to eggs of Tuta absoluta on tomato. Insects, v. 7, p. 1-10, 2016.

VIVAS, L. E.; CERMELI, M.; GODOY, F. Primera cita de Trigonotylus tenuis Reuter, 1893 (Hemiptera: Miridae) causando daños en el cultivo del arroz (Oryza sativa L.) en Venezuela. Entomotropica, v. 20, p. 125-126, 2005.

WHEELER, A. G. Biology of the Plant Bugs (Hemiptera: Miridae). Pests, predators, opportunists, Cornell University Press, Ithaca and London, UK, 2001.

ZAWADNEAK, M. A. C.; GONÇALVES, R. B.; PIMENTEL, I. C.; SCHUBER, J. M.; SANTOS, B.; POLTRONIERI, A. S.; SOLIS, M. A. First record of Duponchelia fovealis (Lepidoptera: Crambidae) in South America. Idesia (Arica), v. 34, p. 91-95, 2016.

Recebido em: 11/04/2019

Aprovado em: 05/08/2019 